University of South Florida

DIGITAL COMMONS

@ UNIVERSITY OF SOUTH FLORIDA
Digital Commons @ University of

South Florida

8-1-2012

\title{
Analysis of the Status and Impacts of NCTR Projects
}

CUTR

Follow this and additional works at: https://digitalcommons.usf.edu/cutr_nctr

\section{Recommended Citation}

"Analysis of the Status and Impacts of NCTR Projects," National Center for Transit Research (NCTR) Report No. CUTR-NCTR-RR-2011-02, Center for Urban Transportation Research, University of South Florida, 2012.

DOI: https://doi.org/10.5038/CUTR-NCTR-RR-2011-02

Available at: https://scholarcommons.usf.edu/cutr_nctr/114

This Technical Report is brought to you for free and open access by the National Center for Transit Research (NCTR) Archive (2000-2020) at Digital Commons @ University of South Florida. It has been accepted for inclusion in Research Reports by an authorized administrator of Digital Commons @ University of South Florida. For more information, please contact digitalcommons@usf.edu. 

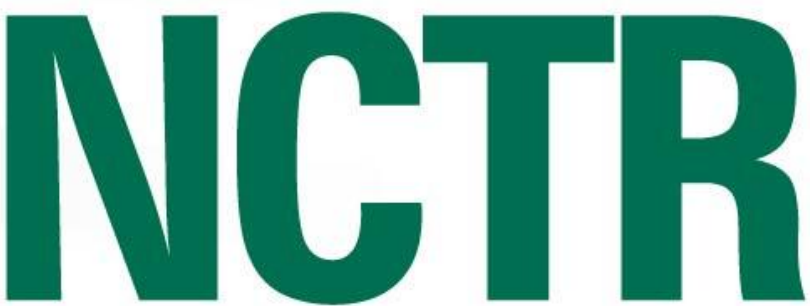

NATIONAL CENTER for TRANSIT RESEARCH

\section{Analysis of the Status and Impacts of NCTR Projects}

August 2012

PROJECT NO. FDOT BDK85 \#977-30

PREPARED FOR

Florida Department of Transportation

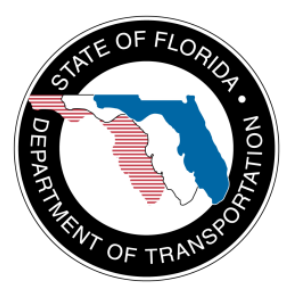




\section{Disclaimer}

The contents of this report reflect the views of the authors, who are responsible for the facts and the accuracy of the information presented herein. This document is disseminated under the sponsorship of the Department of Transportation University Transportation Centers Program and the Florida Department of Transportation, in the interest of information exchange. The U.S. Government and the Florida Department of Transportation assume no liability for the contents or use thereof.

The opinions, findings, and conclusions expressed in this publication are those of the authors and not necessarily those of the State of Florida Department of Transportation. 


\title{
Analysis of the Status and Impacts of NCTR Projects
}

\author{
Final Report
}

Prepared for

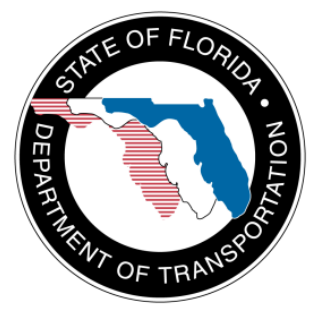

Florida Department of Transportation

605 Suwannee Street, MS 30

Tallahassee, Florida 32399-0450

Project Manager: Mark Greeley

Prepared by

Michael J. Audino

\author{
NCTR \\ National Center for Transit Research \\ Center for Urban Transportation Research (CUTR) \\ University of South Florida \\ 4202 East Fowler Avenue, CUT100 \\ Tampa, Florida 33620-5375
}

August 2012

BDK85 977-30 


\section{Metric Conversion}

\begin{tabular}{|c|c|c|c|c|}
\hline SYMBOL & $\begin{array}{l}\text { WHEN YOU } \\
\text { KNOW }\end{array}$ & MULTIPLY BY & TO FIND & SYMBOL \\
\hline \multicolumn{5}{|c|}{ LENGTH } \\
\hline in & inches & 25.4 & millimeters & $\mathrm{mm}$ \\
\hline ft & feet & 0.305 & meters & $\mathrm{m}$ \\
\hline yd & yards & 0.914 & meters & $\mathrm{m}$ \\
\hline mi & miles & 1.61 & kilometers & $\mathrm{km}$ \\
\hline \multicolumn{5}{|c|}{ VOLUME } \\
\hline floz & fluid ounces & 29.57 & milliliters & $\mathrm{mL}$ \\
\hline gal & gallons & 3.785 & liters & $\mathrm{L}$ \\
\hline $\mathrm{ft}^{3}$ & cubic feet & 0.028 & cubic meters & $\mathrm{m}^{3}$ \\
\hline$y d^{3}$ & cubic yards & 0.765 & cubic meters & $\mathrm{m}^{3}$ \\
\hline \multicolumn{5}{|c|}{ NOTE: volumes greater than $1000 \mathrm{~L}$ shall be shown in $\mathrm{m}^{3}$} \\
\hline \multicolumn{5}{|c|}{ MASS } \\
\hline $\mathbf{O z}$ & ounces & 28.35 & grams & $g$ \\
\hline lb & pounds & 0.454 & kilograms & $\mathrm{kg}$ \\
\hline $\mathbf{T}$ & short tons (2000 lb) & 0.907 & $\begin{array}{c}\text { megagrams } \\
\text { (or "metric } \\
\text { ton") }\end{array}$ & $\mathrm{Mg}($ or "t") \\
\hline \multicolumn{5}{|c|}{ TEMPERATURE (exact degrees) } \\
\hline${ }^{\circ} \mathbf{F}$ & Fahrenheit & $\begin{array}{c}5(F-32) / 9 \\
\text { or }(F-32) / 1.8\end{array}$ & Celsius & ${ }^{\circ} \mathrm{C}$ \\
\hline
\end{tabular}


TECHNICAL REPORT STANDARD TITLE PAGE

\begin{tabular}{|c|c|c|c|}
\hline $\begin{array}{l}\text { 1. Report No. } \\
\text { BDK85 977-30 }\end{array}$ & 2. Government Accession No. & \multicolumn{2}{|c|}{ 3. Recipient's Catalog No. } \\
\hline \multirow{2}{*}{\multicolumn{2}{|c|}{$\begin{array}{l}\text { 4. Title and Subtitle } \\
\text { Analysis of the Status and Impacts of NCTR Reports }\end{array}$}} & \multicolumn{2}{|l|}{$\begin{array}{l}\text { 5. Report Date } \\
\text { August } 2012\end{array}$} \\
\hline & & \multicolumn{2}{|c|}{ 6. Performing Organization Code } \\
\hline \multicolumn{2}{|l|}{$\begin{array}{l}\text { 7. Author(s) } \\
\text { Michael J. Audino }\end{array}$} & \multicolumn{2}{|c|}{$\begin{array}{l}\text { 8. Performing Organization Report No. } \\
\text { NCTR 77934/BDK85 977-30 } \\
\text { U.S.DOT DTRS98-G-0032 }\end{array}$} \\
\hline \multirow{2}{*}{\multicolumn{2}{|c|}{$\begin{array}{l}\text { 9. Performing Organization Name and Address } \\
\text { National Center for Transit Research } \\
\text { Center for Urban Transportation Research (CUTR) } \\
\text { University of South Florida } \\
4202 \text { East Fowler Avenue, CUT100, Tampa, FL 33620-5375 }\end{array}$}} & \multicolumn{2}{|c|}{ 10. Work Unit No. (TRAIS) } \\
\hline & & \multicolumn{2}{|c|}{$\begin{array}{l}\text { 11. Contract or Grant No. } \\
\text { BDK85 } 977-30\end{array}$} \\
\hline \multicolumn{2}{|c|}{$\begin{array}{l}\text { 12. Sponsoring Agency Name and Address } \\
\text { Florida Department of Transportation } \\
\text { Research Center } \\
605 \text { Suwannee Street, MS30 } \\
\text { Tallahassee, Florida } 32399\end{array}$} & \multicolumn{2}{|c|}{$\begin{array}{l}\text { 13. Type of Report and Period Covered } \\
\text { Final } 2 / 3 / 2011-8 / 31 / 2012\end{array}$} \\
\hline \multirow{2}{*}{\multicolumn{4}{|c|}{$\begin{array}{l}\text { Research and Innovative Technology Administration } \\
\text { U.S. Department of Transportation } \\
\text { Mail Code RDT-30, } 1200 \text { New Jersey Ave, SE, Room E33 } \\
\text { Washington, D.C. } 20590-0001 \\
15 \text { Sunplementary Notes }\end{array}$}} \\
\hline & & & \\
\hline \multicolumn{4}{|c|}{$\begin{array}{l}\text { 16. Abstract } \\
\text { The National Center for Transit Research (NCTR) at the University of South Florida (USF) assessed the } \\
\text { implementation status and identified the outcomes and impacts of the results of } 30 \text { Florida Department of } \\
\text { Transportation -sponsored NCTR research projects that concluded in fiscal years } 2008-2010 \text {. The study } \\
\text { attempted to address three primary research questions: } 1 \text { ) How well do research results reach the } \\
\text { customer? 2) How are recipients of the research findings putting the research into practice? 3) If } \\
\text { research is put into practice, what impacts are realized? The report includes a brief summary of each of } 30 \\
\text { NCTR-funded research projects, a summary of the research findings, a status update, and, where } \\
\text { possible, a discussion of the impacts of each project. The report concludes with a discussion of process } \\
\text { improvements that might be implemented to ensure future research projects produce the desired impacts. }\end{array}$} \\
\hline $\begin{array}{l}\text { 17. Key Word } \\
\text { Research findings, knowledge transfer, } \\
\text { research impacts }\end{array}$ & $\begin{array}{l}\text { 18. Distribution Statemen } \\
\text { No Restrictions }\end{array}$ & & \\
\hline $\begin{array}{l}\text { 19. Security Classif. (of this report) } \\
\text { Unclassified }\end{array}$ & $\begin{array}{l}\text { 20. Security Classif. (of this page) } \\
\text { Unclassified }\end{array}$ & $\begin{array}{l}\text { 21. No. of Pages } \\
65\end{array}$ & 22. Price \\
\hline
\end{tabular}

Form DOT F 1700.7 


\section{Executive Summary}

The National Center for Transit Research (NCTR), domiciled at the Center for Urban Transportation Research (CUTR) at the University of South Florida (USF), assessed the implementation status and identified the outcomes and impacts of the results of 30 Florida Department of Transportation (FDOT)-sponsored NCTR research projects that concluded in fiscal years 2008-2010.

The study attempted to answer the following three research questions:

1. How well did the research results reach the customer?

2. How did the customer utilize the research results?

3. How did the research results impact the customer?

Both qualitative and quantitative data were collected. The qualitative data were captured through interviews with each Principal Investigator and, in some cases, other members of the research team. Additional qualitative data were captured via interviews with individuals who were either associated with the research and/or who were involved with utilization of the research results.

In some instances, the qualitative data helped provide answers to research questions two and three. The qualitative data also revealed wide variation with respect to project awareness and project utilization. In other instances, the qualitative data collection process failed to provide answers to the research questions, primarily due to the difficulty of identifying and communicating with users of the research.

Quantitative data were obtained via a Web-based consumer survey, a search of the Google Scholars' data base, and an analysis of research report utilization rates from NCTR's website. These data did not directly provide answers to the research questions, but did offer indirect evidence, particularly to research question one.

The information collected was analyzed and is summarized and organized throughout the body of this report to provide the reader a perspective on the current status and impact of each of the 30 research projects.

In some instances, the data provided answers to the research questions and revealed high levels of customer awareness and specific examples of positive impacts. In other instances, the data collection failed to provide answers to the research questions. This "failure" was primarily a function of the nature of the research and the degree to which results were promoted throughout the industry.

The information presented in succeeding sections of this report includes a brief summary of each of the 30 NCTR-funded research projects, a summary of the research findings, a status update, and a discussion of the impacts of each project. 
This research initiative also provides an informational feedback loop for FDOT that will help FDOT and NCTR better understand what types of research projects tend to produce the most widespread impacts. The report concludes with a discussion of process improvements that might be implemented to ensure that future research projects produce the desired impacts.

\section{Recommendations Summary}

Input from NCTR Principal Investigators and third party stakeholder interviews was assessed to develop process improvement recommendations that could improve NCTR's ability to ensure research project results reach the targeted audiences. Preliminary recommendations were discussed with a three member team of NCTR researchers in advance of developing the final list. The final recommendations centered around four broad areas: the role of NCTR's management team, the capacity of NCTR researchers, the NCTR research project process, and the role of the Florida Department of Transportation Research Office.

The recommendations are discussed in more detail in Chapter 5.

1. Add a "research results outreach and dissemination" task to NCTR project schedules that commences upon final report approval.

2. Provide marketing/public outreach training to Principal Investigators.

3. NCTR's management team should assume a greater leadership role for ensuring that research project results reach targeted audiences. NCTR's management team should meet annually with each Principal Investigator and communicate the management team's expectations for outreach and results dissemination.

4. Include an element in each Quarterly Progress Report that specifies actions and planned activities the Principal Investigator AND the FDOT Project Manager have and will implement that focus on outreach and results dissemination.

5. Continue the current trend for Principal Investigators to utilize webinars as a means to share research results. Incorporate social media as a tactic for sharing research results.

6. Better inform internal customers (CUTR employees) of the outcomes and products of NCTR projects.

7. Principal Investigators and the NCTR leadership team should identify and/or create training and education opportunities outside Florida.

8. NCTR should identify and utilize non-traditional sources for publishing and posting NCTR research results and findings.

9. Establish a technology transfer mindset among CUTR researchers.

10. Retain a professional technology transfer specialist whose role is focused on technology transfer and working with Principal Investigators to bring research products to market. 
11. Revise the CUTR career path process to better incorporate technology transfer activities and successes.

12. Expand professional development/build research capacity of students and younger researchers.

13. CUTR should consider ways to ask those who download reports to contact CUTR and let the researcher know how they are using the information from the report.

14. Diversify the technology transfer product offerings. Not everyone will require the full report nor will everyone benefit from a webinar.

15. Make sure all NCTR projects are listed in Progress and FTA's Research Hub. 


\section{Table of Contents}

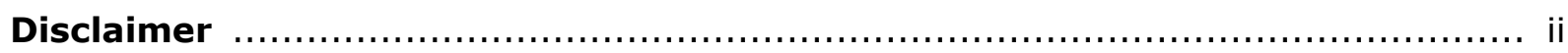

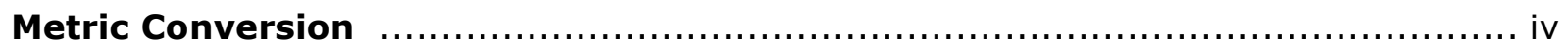

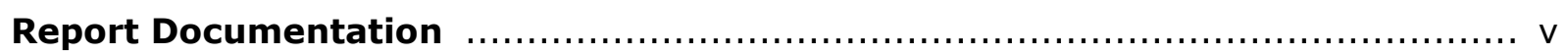

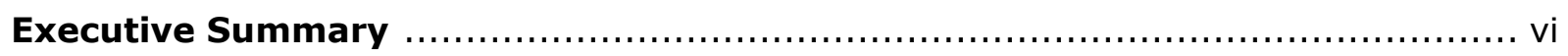

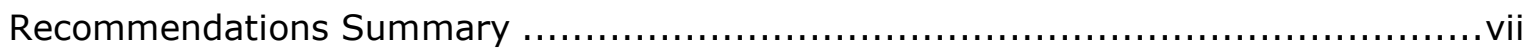

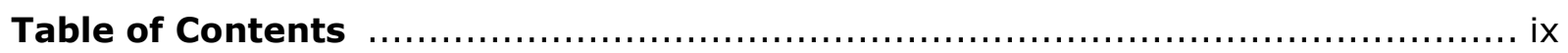

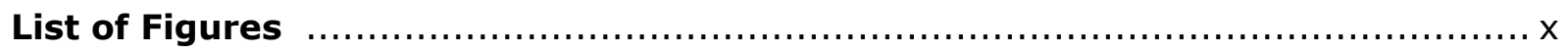

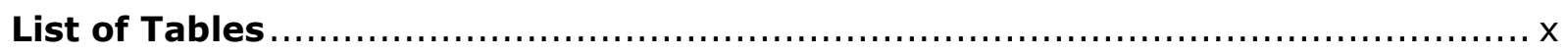

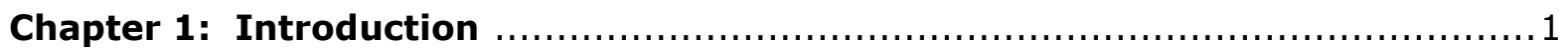

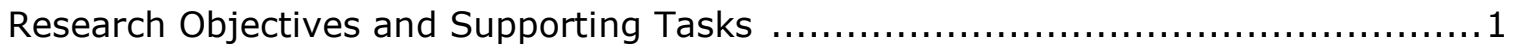

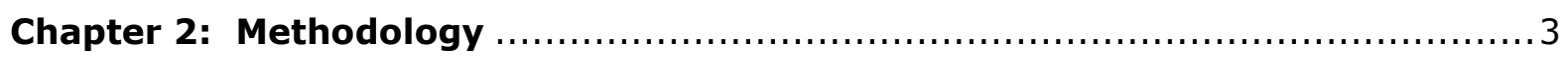

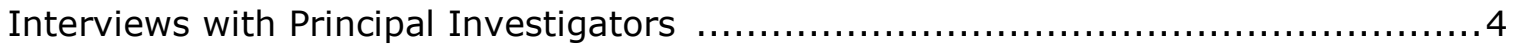

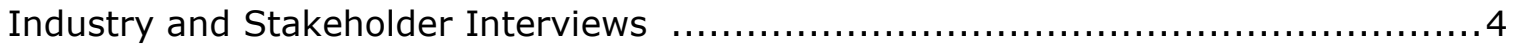

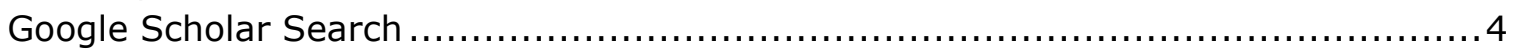

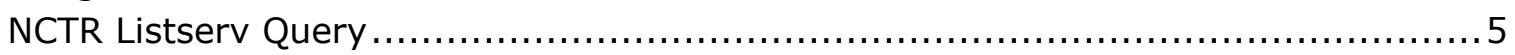

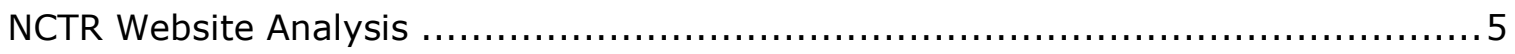

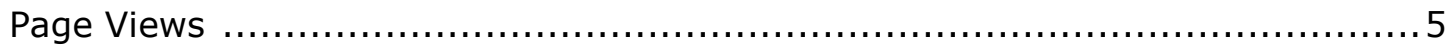

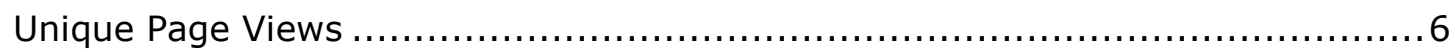

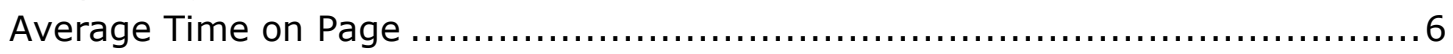

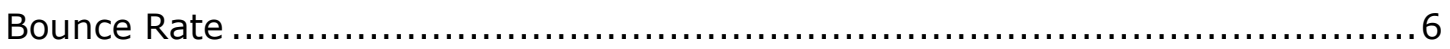

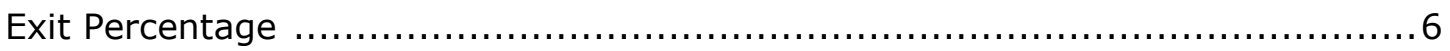

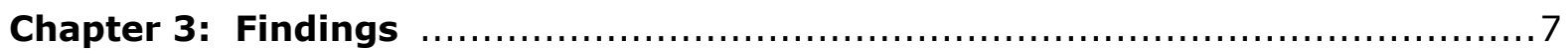

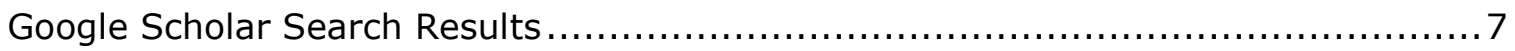

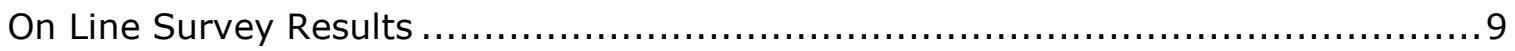

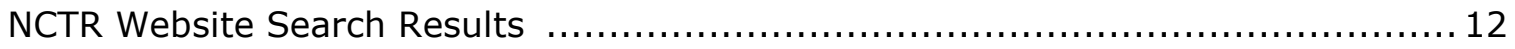

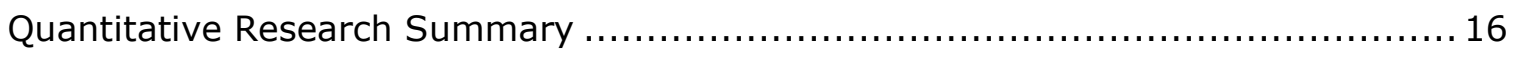

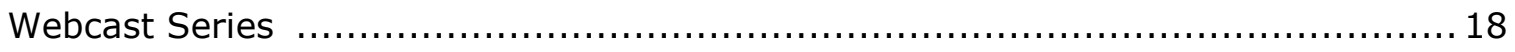

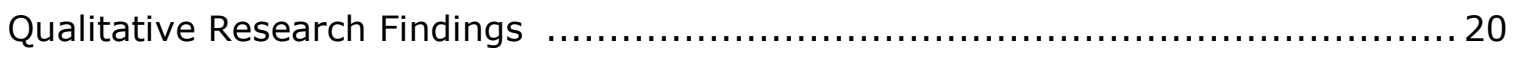

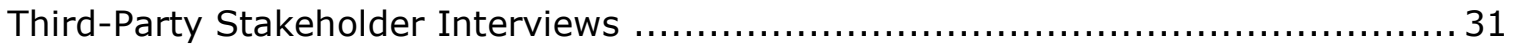

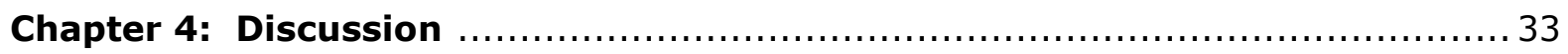

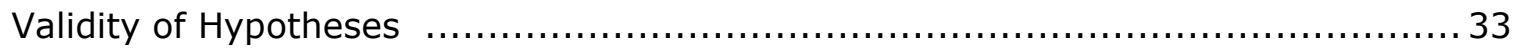

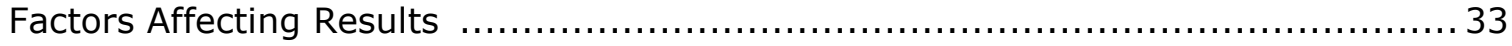

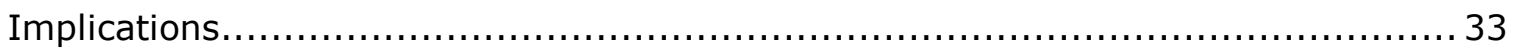




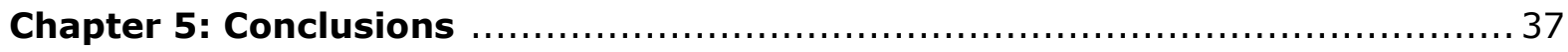

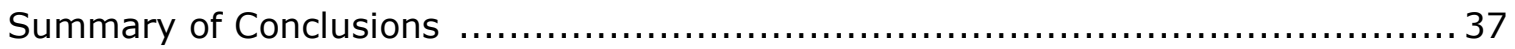

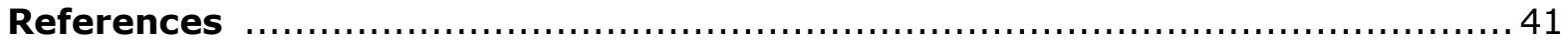

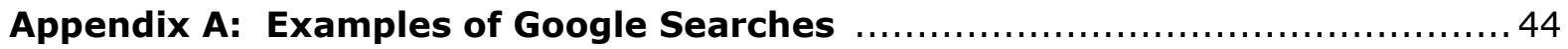

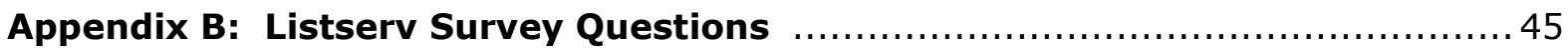

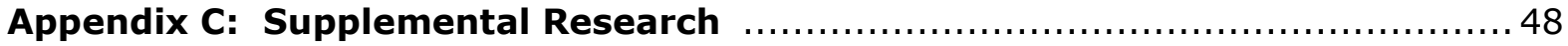

\section{List of Figures}

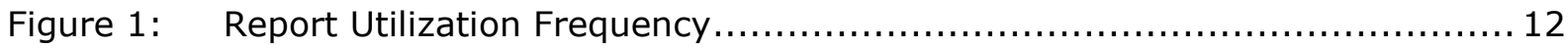

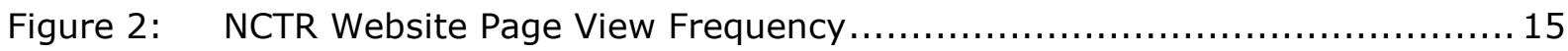

Figure A.1: Representative Screen Shot - Google Scholar Search ........................... 44

Figure A.2: Representative Search Results Page - Google Scholar ..........................4 44

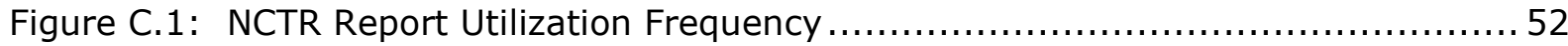

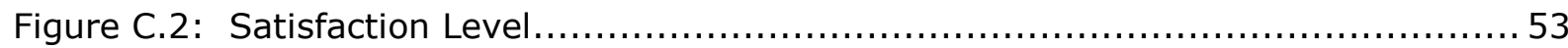

\section{List of Tables}

Table 1: Google Scholars Search Summary ................................................. 7

Table 2: $\quad$ Listserv Summary Results.............................................................. 9

Table 3: $\quad$ NCTR Website Search Results........................................................ 12

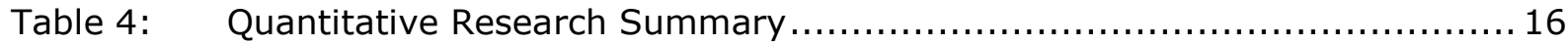

Table B.1: Responses to Open Ended Question - Listserv Survey .......................... 45

Table C.1: Email Survey Summary Results .................................................. 49 


\section{Chapter 1 \\ Introduction}

The National Center for Transit Research (NCTR), domiciled at the Center for Urban Transportation Research (CUTR) at the University of South Florida, conducts applied and advanced research, working closely with metropolitan planning organizations (MPOs), transit organizations, policy makers, departments of transportation, and other key stakeholders within the public transportation industry. The Florida Department of Transportation recognizes NCTR's value in these areas and funds NCTR projects that benefit residents of Florida and public transportation stakeholders across the country and around the globe.

Both the Florida Department of Transportation's Research Center and NCTR recognize the criticality of ensuring the results derived from research projects are reaching the intended customer and are having meaningful impacts on the transit industry, particularly in Florida. Additionally, both parties strive to apply the principles of continuous quality improvement, with particular emphasis on satisfying customer need and maximizing customer value, constantly adjusting and improving business methods to achieve these goals.

\section{Research Objectives and Supporting Tasks}

In this study, NCTR assessed the implementation status and identified the outcomes and impacts of the results of the 30 FDOT-sponsored NCTR research projects that concluded in fiscal years 2008-2010. The goal of this study was to answer three primary research questions:

1. How well did the research results reach the customer?

2. How did the customer utilize the research results?

3. How did the research results impact the customer?

The study assessed the implementation status of each research project, the level of customer awareness of each research project, and attempted to identify specific impacts of each research project. The research was supported through the collection of both qualitative and quantitative data such as: citations in professional journals, page views from the NCTR website, patent applications, and publications generated from project work.

The information collected was analyzed, summarized, and organized to provide an accurate perspective on the current state of the 30 targeted research projects. The information contained in succeeding chapters includes a brief summary of each of the 30 research projects, a brief listing of the research findings, an implementation status, and, where possible, a discussion of the impacts of each project.

This research initiative provides an informational feedback loop - via observations and recommendations - which will help FDOT and NCTR better understand what types of 
research projects tend to produce the most widespread impacts and what process improvements might be implemented to ensure future research projects produce the desired impacts.

Simply stated, some projects were more difficult than others to document in terms of their overall utilization and ultimate impact. The type of research conducted through NCTR is intended to benefit the entire public transportation community, not an individual client. Hence, tracking those who have used the research presented multiple challenges, since it could be used by dozens or even hundreds of agencies. In spite of these challenges, NCTR understands the need to document that the public transportation community and the State of Florida are indeed benefiting from the research being funded by FDOT through NCTR. 


\section{Chapter 2 \\ Methodology}

NCTR initiated the research with a project kick-off meeting with the FDOT project manager, which ensured both parties clearly understood the purpose of the research, the proposed research tasks, schedule, milestones, deliverables, reporting requirements, and deployment plan.

The author reviewed the scope of work for each of the 30 NCTR projects to identify the initial reason(s) for conducting the project and to identify the targeted audience of the research results. This information assisted the author in determining if the intended customers received the research results, if the intended customers utilized the research results, and how the research results impacted the intended customers.

Each NCTR Principal Investigator (PI) who is currently employed at CUTR was asked to provide information on what agencies they worked with (if any) as they completed their project. They were also asked to provide any records (emails, letters, and notes) that demonstrated how their project had positively impacted targeted customers. Each PI was asked to determine if agencies that have utilized the findings of their research could identify and quantify any changes (cost savings, safety improvements, policy changes, operational procedures, etc.) that resulted from instituting practices due to the research project's findings and outcomes. In addition, each PI was asked to produce correspondence they had received requesting more information on the subject of the report.

To varying degrees, each PI provided documentation of published papers they produced that were part of professional proceedings, as well as a record of the presentations they made of the findings of their research at professional conferences.

A Google Scholar search was conducted to identify how frequently each of the 30 research reports had been referenced in professional journals and articles.

To ensure sufficient outreach and input, an online survey was disseminated to over 3,400 registrants of a variety of CUTR and NCTR-sponsored listservs. The author contacted the online survey respondents who had expressed a willingness to discuss the survey responses in more detail.

The NCTR website was analyzed to determine the number of hits and downloads that each of the 30 projects had experienced.

The author interviewed each project PI to assess their unique perspectives on project implementation, clarify any of the information and data previously collected, identify industry stakeholders who may have utilized the research results, and identify and documented any new software and utilization of that software or technology by others. 
To the extent possible, the author interviewed stakeholders identified by the PIs to discuss how the stakeholders may have utilized the research results, determine specific actions taken as a result of the research project's findings, and assess how application of research findings positively impacted the individual stakeholder and/or their organization. If the research produced any new software or technology, the author identified and documented its utilization.

Based on these activities, the author produced a series of process improvement recommendations.

\section{Google Scholar Search}

CUTR conducted a systematic search of the 30 NCTR projects using Google Scholar. Using the Google Scholar source, the number of times the projects were cited by other articles and publications was noted. In addition, the projects that were cached in their respective journals were also identified.

In Google Scholar, the Advanced Scholar Search allows users to search for a research paper/publication using the name, author, publication, date and/or collections. Appendix A provides a screen shot of an active search in progress. Google Scholar then performs a comprehensive search in all of its resources and displays results. The main results are basically links to the paper/publication that was entered in the Advanced Scholar Search window. Google Scholar also identifies the frequency of citations from other publications/papers. For example, NCTR Research Report "Smart Phone Application to Influence Travel Behavior (TRAC-IT Phase 3)" by Sean Barbeau had 8 citations.

\section{NCTR Listserv Query}

In early summer 2011, the author developed a survey instrument utilizing SurveyMonkey to gauge the utilization and benefit of NCTR's research by those stakeholders who are active members of NCTR and CUTR listservs. The survey instrument included the following four questions:

- Question 1. Please indicate if you or your organization have utilized the findings or applied the tool from any of the following research projects conducted by the National Center for Transit Research at the University of South Florida.

- Question 2. Please describe how you used the results and/or tools for those projects you checked "Yes" above (Question 1).

- Question 3. What specific suggestions do you have for improving the manner in which NCTR research results and tools are made public or made available to you?

- Question 4. If you answered YES to any of the questions and if you are willing to discuss your responses with us, PLEASE provide your name, email and telephone number 
On July 22, 2011, the online survey was launched. The link to the survey was provided by e-mail to 30 employees of the Florida Department of Transportation and to over 3,400 transportation professionals, each of whom was a registered member of one or more listservs managed by CUTR and/or NCTR staff. The listservs included:

- CUTR

- Journal of Public Transportation

- RTAP

- TRANSP-TDM

- $\quad$ BRT

- BFM-General (bus fleet maintenance)

- Leadership APTA

- Florida Public Transit Association

- Florida Transit Marketing Network

The direct e-mail recipients of the survey link were encouraged to forward it to colleagues, and so the total number of unique email recipients is unknown. As a supplemental task, a similar survey instrument was utilized to generate the findings discussed in Appendix C.

\section{NCTR Website Analysis}

During this phase a systematic data collection effort of NCTR projects was conducted using Google Analytics. The following definitions are excerpted from the Google website (www.google.com) and explain the standard nomenclature utilized by Google Analytics.

Page Views - the total number of pages viewed on the site and is a general measure of how extensively the site is used. It is more useful as a basic indicator of the traffic load on the site and server than as a marketing measure.

Unique Page Views - the total number of page views, by each user. The same user who might visit the page multiple times would contribute to the page views every time she/he visits the page, but would contribute only once to the unique page views.

Average Time on Page - one way of measuring visit quality. If visitors spend a long time visiting the page, they may be interacting extensively with it. However, Average Time on Page can be misleading because visitors often leave browser windows open when they are not actually viewing or using the page.

Bounce Rate - the percentage of visits to a website in which a visitor views only one page and leaves. Bounce rate is a measure of visitor interaction, and a high bounce rate generally indicates that the site page was not relevant to the visitor. Bounce rates can be minimized by tailoring pages to potential visitor's interests by better internal keywording (a Web programming aspect) and by more specificity in advertisements for the pages. Pages should provide the information and services that were promised in the advertisement copy. 
Exit Percentage - the Number of Exits identifies the number of exits from the site, and, as with entrances, it will always be equal to the number of visits when applied over the entire website. The "Exit \%" is the percentage of site exits that occurred from a page or set of pages.

\section{Interviews with Principal Investigators}

A Principal Investigator directed each of the 30 NCTR projects analyzed. Several projects had multiple investigators. Each investigator was contacted via e-mail and advised of the purpose and intent of the Analysis of the Status and Impacts of NCTR Projects research initiative. Subsequently, personal meetings were conducted with each Principal Investigator and, on occasions, members of the research team.

Each interview followed a similar format. Interviewees validated the status of the research project; identified the target customer base for the research product; discussed the research findings; identified research papers they had published and professional presentations they had delivered, along with any shared feedback on the research from professional colleagues; and identified potential third-party stakeholders with whom the interviewer could speak.

\section{Industry and Stakeholder Interviews}

For some projects, the project's Principal Investigator was able to identify individuals who were instrumental in providing guidance on the research, utilized the research findings in some way, and/or helped share research findings with others. Where these third party stakeholders were identified, the author attempted to conduct follow-up interviews and gain additional insight into the outreach and technology transfer process. In many cases, the author's attempts to contact third party stakeholders proved unsuccessful.

\section{Supplemental Research}

At the completion of this research project it was decided to distribute an additional online survey to high level public transportation officials in Florida. The survey asked them to indicate if their organization had utilized the research findings in some way, and/or helped share research findings with others. 


\section{Chapter 3 \\ Findings}

\section{Google Scholar Search Results}

Table 1 summarizes the citation frequency from the Google Scholar search for each of the 30 targeted NCTR projects. The search was conducted in August 2011. Projects are listed first by frequency of citation (in descending order) and then by year of publication (in descending order). A higher number of citations is a positive indicator of a report's utilization by others and the implied benefit of the research to others.

Table 1: Google Scholars Search Summary

\begin{tabular}{|c|c|c|c|c|c|}
\hline & Title & Author & Project & $\begin{array}{c}\text { Year } \\
\text { Published }\end{array}$ & Citations \\
\hline 1 & $\begin{array}{l}\text { Smart Phone Application to Influence } \\
\text { Travel Behavior (TRAC-IT Phase 3) }\end{array}$ & Barbeau & BD549-35 & 2008 & 8 \\
\hline 2 & $\begin{array}{l}\text { Guidebook on Using American Community } \\
\text { Survey Data for Transit Planning }\end{array}$ & Chu & $\begin{array}{l}\text { BDK85977- } \\
\quad 02\end{array}$ & 2005 & 8 \\
\hline 3 & $\begin{array}{l}\text { Travel Assistance Device (TAD) to Aid } \\
\text { Transit Riders with Special Needs }\end{array}$ & Barbeau & BD549-33 & 2008 & 5 \\
\hline 4 & $\begin{array}{l}\text { Best Practices In Transit Services } \\
\text { Planning }\end{array}$ & Goodwill & BD549-38 & 2009 & 2 \\
\hline 5 & $\begin{array}{l}\text { Exploration of a Shift in Household } \\
\text { Transportation Spending from Vehicles to } \\
\text { Public Transportation }\end{array}$ & Polzin & BD549-43 & 2008 & 2 \\
\hline 6 & $\begin{array}{l}\text { Transit Ridership, Reliability and } \\
\text { Retention }\end{array}$ & Perk & BD549-32 & 2008 & 2 \\
\hline 7 & $\begin{array}{l}\text { Transit Extraboard Management- } \\
\text { Optimum Sizing and Strategies }\end{array}$ & DeAnnuntis & BD549-23 & 2008 & 2 \\
\hline 8 & $\begin{array}{l}\text { Synthesis of Research on Value of Time } \\
\text { and Value of Reliability }\end{array}$ & Concas & BD549-46 & 2009 & 1 \\
\hline 9 & $\begin{array}{l}\text { Development of Comprehensive Guidance } \\
\text { on Obtaining Service Consumed Data for } \\
\text { National Transit Database (NTD) }\end{array}$ & Chu & BD549-47 & 2009 & 1 \\
\hline 10 & $\begin{array}{l}\text { Evaluation of Smart Video for Transit } \\
\text { Event Detection }\end{array}$ & Sapper & BD549-49 & 2009 & 1 \\
\hline 11 & $\begin{array}{l}\text { Evaluation of Electronic Data Recorder for } \\
\text { Incident Investigation, Driver } \\
\text { Performance and Vehicle Maintenance }\end{array}$ & Sapper & BD549-50 & 2009 & 1 \\
\hline 12 & $\begin{array}{l}\text { Quantifying Net Social Benefits of Vehicle } \\
\text { Trip Reduction Impacts to make Existing } \\
\text { Road Infrastructure Perform Better- } \\
\text { Guidance for Customizing the TRIMMS } \\
\text { Model to Aid Local, Regional and State } \\
\text { Decision Makers }\end{array}$ & Concas & BD549-52 & 2009 & 1 \\
\hline
\end{tabular}


Table 1: Google Scholars Search Summary (cont'd)

\begin{tabular}{|c|c|c|c|c|c|}
\hline & Title & Author & Project & $\begin{array}{c}\text { Year } \\
\text { Published }\end{array}$ & Citations \\
\hline 13 & $\begin{array}{l}\text { Utilizing Information Technology in } \\
\text { Innovative Marketing Approaches for } \\
\text { Public Transportation }\end{array}$ & Morris & BD549-53 & 2009 & 1 \\
\hline 14 & $\begin{array}{l}\text { Testing the Impact of Personalized } \\
\text { Feedback on Household Travel Behavior }\end{array}$ & Winters & BD549-24 & 2008 & 1 \\
\hline 15 & $\begin{array}{l}\text { Guidelines and Performance Measures to } \\
\text { Incorporate Transit and Other Multimodal } \\
\text { Considerations into the FDOT DRI Review } \\
\text { Process }\end{array}$ & Seggerman & BD549-31 & 2008 & 1 \\
\hline 16 & $\begin{array}{l}\text { Programs that Match Seniors with } \\
\text { Volunteer Drivers }\end{array}$ & Hendricks & BD549-41 & 2008 & 1 \\
\hline 17 & $\begin{array}{l}\text { Developing a Printed Transit Information } \\
\text { Material Design Manual }\end{array}$ & Cain & BD549-29 & 2007 & 1 \\
\hline 18 & Guidebook for Start-up Transit Agencies & Goodwill & BD549-14 & 2006 & 1 \\
\hline 19 & $\begin{array}{l}\text { Developing a Technique that Predicts the } \\
\text { Impacts of TDM on a Transportation } \\
\text { System }\end{array}$ & Georggi & $\begin{array}{l}\text { BDK85977- } \\
\quad 06\end{array}$ & 2010 & 0 \\
\hline 20 & $\begin{array}{l}\text { Evaluation of Camera Based Systems to } \\
\text { Reduce Transit Bus Side Collisions }\end{array}$ & Lin & $\begin{array}{l}\text { BDK85977- } \\
08\end{array}$ & 2010 & 0 \\
\hline 21 & $\begin{array}{l}\text { Investigation of the Feasibility of Toll and } \\
\text { Transit Agency Equity Sharing }\end{array}$ & Reich & $\begin{array}{l}\text { BDK85977- } \\
09\end{array}$ & 2010 & 0 \\
\hline 22 & $\begin{array}{l}\text { Regional Fare Policy and Fare Allocation, } \\
\text { Innovations in Fare Equipment and Data } \\
\text { Collection }\end{array}$ & Joslin & BD549-51 & 2010 & 0 \\
\hline 23 & $\begin{array}{l}\text { Creative Ways to Manage Paratransit } \\
\text { Costs }\end{array}$ & Goodwill & BD549-28 & 2008 & 0 \\
\hline 24 & Integrating Transit and Urban Form & Concas & BD549-37 & 2008 & 0 \\
\hline 25 & $\begin{array}{l}\text { Development of Large Bus/Small Bus } \\
\text { Decision Support Tool }\end{array}$ & Reich & BD549-39 & 2008 & 0 \\
\hline 26 & $\begin{array}{l}\text { Development of NTD Tool for Vanpool } \\
\text { Services }\end{array}$ & Chu & BD549-40 & 2008 & 0 \\
\hline 27 & $\begin{array}{l}\text { Impacts of More Rigorous ADA Paratransit } \\
\text { Eligibility Assessments on Riders with } \\
\text { Disabilities }\end{array}$ & Sapper & BD549-44 & 2008 & 0 \\
\hline 28 & $\begin{array}{l}\text { Enhancing Transit Safety and Security } \\
\text { with Wireless Detection and } \\
\text { Communication Technologies }\end{array}$ & Barbeau & BD549-45 & 2008 & 0 \\
\hline 29 & Toolbox for Transit Event Investigation & Sapper & BD549-22 & 2007 & 0 \\
\hline 30 & Moving the Bus Back Into Traffic Safety & Lin & BD549-34 & 2007 & - \\
\hline
\end{tabular}

The number of citations ranged from 0 to 8. Two research reports, "Guidebook on Using American Community Survey Data for Transit Planning" and "Smart Phone Application to Influence Travel Behavior (TRAC-IT Phase 3)," were cited eight times. The average number of citations was 1.33 . Twelve of the 30 NCTR projects (40\%) were not cited. 


\section{Online Survey Results}

A four-question, online survey was disseminated to over 3,400 registrants of a variety of CUTR and NCTR-sponsored Listservs. The survey was designed to help measure how well the findings and tools from the 30 NCTR projects were known throughout the public transit industry, assess how the findings and tools had been utilized, and identify specific outcomes from the utilization of these findings and tools. A total of 117 survey responses were received, although not all respondents answered every question.

Question 1 provided a listing of the 30 NCTR projects and afforded respondents the opportunity to indicate-for each project-if they or their organization had utilized the findings or applied the tool from the research. Respondents could answer "yes," "no," or "don't know." The percentage of respondents that indicated they had used NCTR research results ranged from 9.2 to 40 percent. One project, Transit Ridership, Reliability and Retention, had the highest utilization rate of 40 percent. The average utilization rate was 20.46 percent. Table 2 provides a summary of the responses to Question 1 of the survey.

Table 2: Listserv Summary Results

\begin{tabular}{|c|c|c|c|c|c|c|c|}
\hline & Title & Author & Project & Yes & No & $\begin{array}{l}\text { Don't } \\
\text { Recall }\end{array}$ & $\begin{array}{l}\text { Response } \\
\text { Count }\end{array}$ \\
\hline 1 & $\begin{array}{l}\text { Smart Phone Application } \\
\text { to Influence Travel } \\
\text { Behavior (TRAC-IT } \\
\text { Phase 3) }\end{array}$ & Barbeau & BD549-35 & $\begin{array}{c}29 \\
(26.6 \%)\end{array}$ & $\begin{array}{c}67 \\
(61.5 \%)\end{array}$ & $\begin{array}{c}13 \\
(11.9 \%)\end{array}$ & 109 \\
\hline 2 & $\begin{array}{l}\text { Guidebook on Using } \\
\text { American Community } \\
\text { Survey Data for Transit } \\
\text { Planning }\end{array}$ & Chu & $\begin{array}{l}\text { BDK85977- } \\
02\end{array}$ & $\begin{array}{c}26 \\
(23.2 \%)\end{array}$ & $\begin{array}{c}63 \\
(56.3 \%)\end{array}$ & $\begin{array}{c}23 \\
(20.5 \%)\end{array}$ & 112 \\
\hline 3 & $\begin{array}{l}\text { Travel Assistance Device } \\
\text { (TAD) to Aid Transit } \\
\text { Riders with Special } \\
\text { Needs }\end{array}$ & Barbeau & BD549-33 & $\begin{array}{c}29 \\
(26.9 \%)\end{array}$ & $\begin{array}{c}69 \\
(63.9 \%)\end{array}$ & $\begin{array}{c}10 \\
(9.3 \%)\end{array}$ & 108 \\
\hline 4 & $\begin{array}{l}\text { Best Practices In Transit } \\
\text { Services Planning }\end{array}$ & Goodwill & BD549-38 & $\begin{array}{c}43 \\
(39.1 \%)\end{array}$ & $\begin{array}{c}56 \\
(50.9 \%)\end{array}$ & $\begin{array}{c}11 \\
(10.0 \%)\end{array}$ & 110 \\
\hline 5 & $\begin{array}{l}\text { Exploration of a Shift in } \\
\text { Household } \\
\text { Transportation Spending } \\
\text { from Vehicles to Public } \\
\text { Transportation }\end{array}$ & Polzin & BD549-43 & $\begin{array}{c}16 \\
(14.8 \%)\end{array}$ & $\begin{array}{c}75 \\
(69.4 \%)\end{array}$ & $\begin{array}{c}17 \\
(15.7 \%)\end{array}$ & 108 \\
\hline 6 & $\begin{array}{l}\text { Transit Ridership, } \\
\text { Reliability and Retention }\end{array}$ & Perk & BD549-32 & $\begin{array}{c}44 \\
(40.0 \%)\end{array}$ & $\begin{array}{c}56 \\
(50.9 \%\end{array}$ & $\begin{array}{c}10 \\
(9.1 \%)\end{array}$ & 110 \\
\hline 7 & $\begin{array}{l}\text { Transit Extraboard } \\
\text { Management-Optimum } \\
\text { Sizing and Strategies }\end{array}$ & DeAnnuntis & BD549-23 & $\begin{array}{c}14 \\
(12.8 \%)\end{array}$ & $\begin{array}{c}78 \\
(71.6 \%)\end{array}$ & $\begin{array}{c}17 \\
(15.6 \%)\end{array}$ & 109 \\
\hline 8 & $\begin{array}{l}\text { Synthesis of Research } \\
\text { on Value of Time and } \\
\text { Value of Reliability }\end{array}$ & Concas & BD549-46 & $\begin{array}{c}16 \\
(14.7 \%)\end{array}$ & $\begin{array}{c}77 \\
(70.6 \%)\end{array}$ & $\begin{array}{c}16 \\
(14.7 \%)\end{array}$ & 109 \\
\hline
\end{tabular}


Table 2: Listserv Summary Results (cont'd)

\begin{tabular}{|c|c|c|c|c|c|c|c|}
\hline & Title & Author & Project & Yes & No & $\begin{array}{l}\text { Don't } \\
\text { Recall }\end{array}$ & $\begin{array}{l}\text { Response } \\
\text { Count }\end{array}$ \\
\hline 9 & $\begin{array}{l}\text { Development of } \\
\text { Comprehensive } \\
\text { Guidance on Obtaining } \\
\text { Service Consumed Data } \\
\text { for National Transit } \\
\text { Database (NTD) }\end{array}$ & Chu & BD549-47 & $\begin{array}{c}10 \\
(9.2 \%)\end{array}$ & $\begin{array}{c}81 \\
(74.3 \%)\end{array}$ & $\begin{array}{c}18 \\
(16.5 \%)\end{array}$ & 109 \\
\hline 10 & $\begin{array}{l}\text { Evaluation of Smart } \\
\text { Video for Transit Event } \\
\text { Detection }\end{array}$ & Sapper & BD549-49 & $\begin{array}{c}12 \\
(11.1 \%)\end{array}$ & $\begin{array}{c}79 \\
(73.1 \%)\end{array}$ & $\begin{array}{c}17 \\
(15.7 \%)\end{array}$ & 108 \\
\hline 11 & $\begin{array}{l}\text { Evaluation of Electronic } \\
\text { Data Recorder for } \\
\text { Incident Investigation, } \\
\text { Driver Performance and } \\
\text { Vehicle Maintenance }\end{array}$ & Sapper & BD549-50 & $\begin{array}{c}15 \\
(13.8 \%)\end{array}$ & $\begin{array}{c}79 \\
(72.5 \%)\end{array}$ & $\begin{array}{c}15 \\
(13.8 \%)\end{array}$ & 109 \\
\hline 12 & $\begin{array}{l}\text { Quantifying Net Social } \\
\text { Benefits of Vehicle Trip } \\
\text { Reduction Impacts to } \\
\text { make Existing Road } \\
\text { Infrastructure Perform } \\
\text { Better-Guidance for } \\
\text { Customizing the } \\
\text { TRIMMS Model to Aid } \\
\text { Local, Regional and } \\
\text { State Decision Makers }\end{array}$ & Concas & BD549-52 & $\begin{array}{c}12 \\
(10.9 \%)\end{array}$ & $\begin{array}{c}78 \\
(70.9 \%)\end{array}$ & $\begin{array}{c}20 \\
(18.2 \%)\end{array}$ & 110 \\
\hline 13 & $\begin{array}{l}\text { Utilizing Information } \\
\text { Technology in } \\
\text { Innovative Marketing } \\
\text { Approaches for Public } \\
\text { Transportation }\end{array}$ & Morris & BD549-53 & $\begin{array}{c}21 \\
(19.1 \%)\end{array}$ & $\begin{array}{c}70 \\
(63.6 \%)\end{array}$ & $\begin{array}{c}19 \\
(17.3 \%)\end{array}$ & 109 \\
\hline 14 & $\begin{array}{l}\text { Testing the Impact of } \\
\text { Personalized Feedback } \\
\text { on Household Travel } \\
\text { Behavior }\end{array}$ & Winters & BD549-24 & $\begin{array}{c}17 \\
(15.5 \%)\end{array}$ & $\begin{array}{c}76 \\
(69.1 \%)\end{array}$ & $\begin{array}{c}17 \\
(15.5 \%)\end{array}$ & 110 \\
\hline 15 & $\begin{array}{l}\text { Guidelines and } \\
\text { Performance Measures } \\
\text { to Incorporate Transit } \\
\text { and Other Multimodal } \\
\text { Considerations into the } \\
\text { FDOT DRI Review } \\
\text { Process }\end{array}$ & Seggerman & BD549-31 & $\begin{array}{c}33 \\
(30.0 \%)\end{array}$ & $\begin{array}{c}63 \\
(57.3 \%)\end{array}$ & $\begin{array}{c}14 \\
(12.7 \%)\end{array}$ & 110 \\
\hline 16 & $\begin{array}{l}\text { Programs that Match } \\
\text { Seniors with Volunteer } \\
\text { Drivers }\end{array}$ & Hendricks & BD549-41 & $\begin{array}{c}16 \\
(14.8 \%)\end{array}$ & $\begin{array}{c}80 \\
(74.1 \%)\end{array}$ & $\begin{array}{c}12 \\
(11.1 \%)\end{array}$ & 108 \\
\hline 17 & $\begin{array}{l}\text { Developing a Printed } \\
\text { Transit Information } \\
\text { Material Design Manual }\end{array}$ & Cain & BD549-29 & $\begin{array}{c}24 \\
(21.8 \%)\end{array}$ & $\begin{array}{c}68 \\
(61.8 \%)\end{array}$ & $\begin{array}{c}18 \\
(16.4 \%)\end{array}$ & 110 \\
\hline
\end{tabular}


Table 2: Listserv Summary Results (cont'd)

\begin{tabular}{|c|c|c|c|c|c|c|c|}
\hline & Title & Author & Project & Yes & No & $\begin{array}{l}\text { Don't } \\
\text { Recall }\end{array}$ & $\begin{array}{l}\text { Response } \\
\text { Count }\end{array}$ \\
\hline 18 & $\begin{array}{l}\text { Guidebook for Start-up } \\
\text { Transit Agencies }\end{array}$ & Goodwill & BD549-14 & $\begin{array}{c}15 \\
(14.0 \%)\end{array}$ & $\begin{array}{c}76 \\
(71.0 \%)\end{array}$ & $\begin{array}{c}16 \\
(15.0 \%)\end{array}$ & 107 \\
\hline 19 & $\begin{array}{l}\text { Developing a Technique } \\
\text { that Predicts the } \\
\text { Impacts of TDM on a } \\
\text { Transportation System }\end{array}$ & Georggi & $\begin{array}{l}\text { BDK85977- } \\
06\end{array}$ & $\begin{array}{c}28 \\
(25.5 \%)\end{array}$ & $\begin{array}{c}64 \\
(58.2 \%)\end{array}$ & $\begin{array}{c}18 \\
(16.4 \%)\end{array}$ & 110 \\
\hline 20 & $\begin{array}{l}\text { Evaluation of Camera } \\
\text { Based Systems to } \\
\text { Reduce Transit Bus Side } \\
\text { Collisions }\end{array}$ & Lin & $\begin{array}{l}\text { BDK85977- } \\
\quad 08\end{array}$ & $\begin{array}{c}23 \\
(20.9 \%)\end{array}$ & $\begin{array}{c}74 \\
(67.3 \%)\end{array}$ & $\begin{array}{c}13 \\
(11.8 \%)\end{array}$ & 110 \\
\hline 21 & $\begin{array}{l}\text { Investigation of the } \\
\text { Feasibility of Toll and } \\
\text { Transit Agency Equity } \\
\text { Sharing }\end{array}$ & Reich & $\begin{array}{l}\text { BDK85977- } \\
09\end{array}$ & $\begin{array}{c}14 \\
(12.8 \%)\end{array}$ & $\begin{array}{c}76 \\
(69.7 \%)\end{array}$ & $\begin{array}{c}19 \\
(17.4 \%)\end{array}$ & 109 \\
\hline 22 & $\begin{array}{l}\text { Regional Fare Policy and } \\
\text { Fare Allocation, } \\
\text { Innovations in Fare } \\
\text { Equipment and Data } \\
\text { Collection }\end{array}$ & Joslin & BD549-51 & $\begin{array}{c}23 \\
(20.9 \%)\end{array}$ & $\begin{array}{c}75 \\
(68.2 \%)\end{array}$ & $\begin{array}{c}12 \\
(10.9 \%)\end{array}$ & 110 \\
\hline 23 & $\begin{array}{l}\text { Creative Ways to } \\
\text { Manage Paratransit } \\
\text { Costs }\end{array}$ & Goodwill & BD549-28 & $\begin{array}{c}25 \\
(22.3 \%)\end{array}$ & $\begin{array}{c}71 \\
(63.4 \%)\end{array}$ & $\begin{array}{c}16 \\
(14.3 \%)\end{array}$ & 112 \\
\hline 24 & $\begin{array}{l}\text { Integrating Transit and } \\
\text { Urban Form }\end{array}$ & Concas & BD549-37 & $\begin{array}{c}29 \\
(27.1 \%)\end{array}$ & $\begin{array}{c}64 \\
(59.8 \%)\end{array}$ & $\begin{array}{c}14 \\
(13.1 \%)\end{array}$ & 107 \\
\hline 25 & $\begin{array}{l}\text { Development of Large } \\
\text { Bus/Small Bus Decision } \\
\text { Support Tool }\end{array}$ & Reich & BD549-39 & $\begin{array}{c}24 \\
(21.8 \%)\end{array}$ & $\begin{array}{c}74 \\
(67.3 \%)\end{array}$ & $\begin{array}{c}12 \\
(10.9 \%)\end{array}$ & 110 \\
\hline 26 & $\begin{array}{l}\text { Development of NTD } \\
\text { Tool for Vanpool } \\
\text { Services }\end{array}$ & Chu & BD549-40 & $\begin{array}{c}16 \\
(14.5 \%)\end{array}$ & $\begin{array}{c}77 \\
(70.0 \%)\end{array}$ & $\begin{array}{c}17 \\
(15.5 \%)\end{array}$ & 110 \\
\hline 27 & $\begin{array}{l}\text { Impacts of More } \\
\text { Rigorous ADA } \\
\text { Paratransit Eligibility } \\
\text { Assessments on Riders } \\
\text { with Disabilities }\end{array}$ & Sapper & BD549-44 & $\begin{array}{c}25 \\
(23.1 \%)\end{array}$ & $\begin{array}{c}71 \\
(65.7 \%)\end{array}$ & $\begin{array}{c}12 \\
(11.1 \%)\end{array}$ & 108 \\
\hline 28 & $\begin{array}{l}\text { Enhancing Transit } \\
\text { Safety and Security with } \\
\text { Wireless Detection and } \\
\text { Communication } \\
\text { Technologies }\end{array}$ & Barbeau & BD549-45 & $\begin{array}{c}19 \\
(17.4 \%)\end{array}$ & $\begin{array}{c}75 \\
(68.8 \%)\end{array}$ & $\begin{array}{c}15 \\
(13.8 \%)\end{array}$ & 109 \\
\hline 29 & $\begin{array}{l}\text { Toolbox for Transit } \\
\text { Event Investigation }\end{array}$ & Sapper & BD549-22 & $\begin{array}{c}20 \\
(18.7 \%)\end{array}$ & $\begin{array}{c}75 \\
(70.1 \%)\end{array}$ & $\begin{array}{c}12 \\
(11.2 \%)\end{array}$ & 107 \\
\hline 30 & $\begin{array}{l}\text { Moving the Bus Back } \\
\text { Into Traffic Safety }\end{array}$ & Lin & BD549-34 & $\begin{array}{c}34 \\
(30.6 \%)\end{array}$ & $\begin{array}{c}66 \\
(59.5 \%)\end{array}$ & $\begin{array}{c}11 \\
(9.9 \%)\end{array}$ & 111 \\
\hline
\end{tabular}


Figure 1 provides a summary of report utilization.

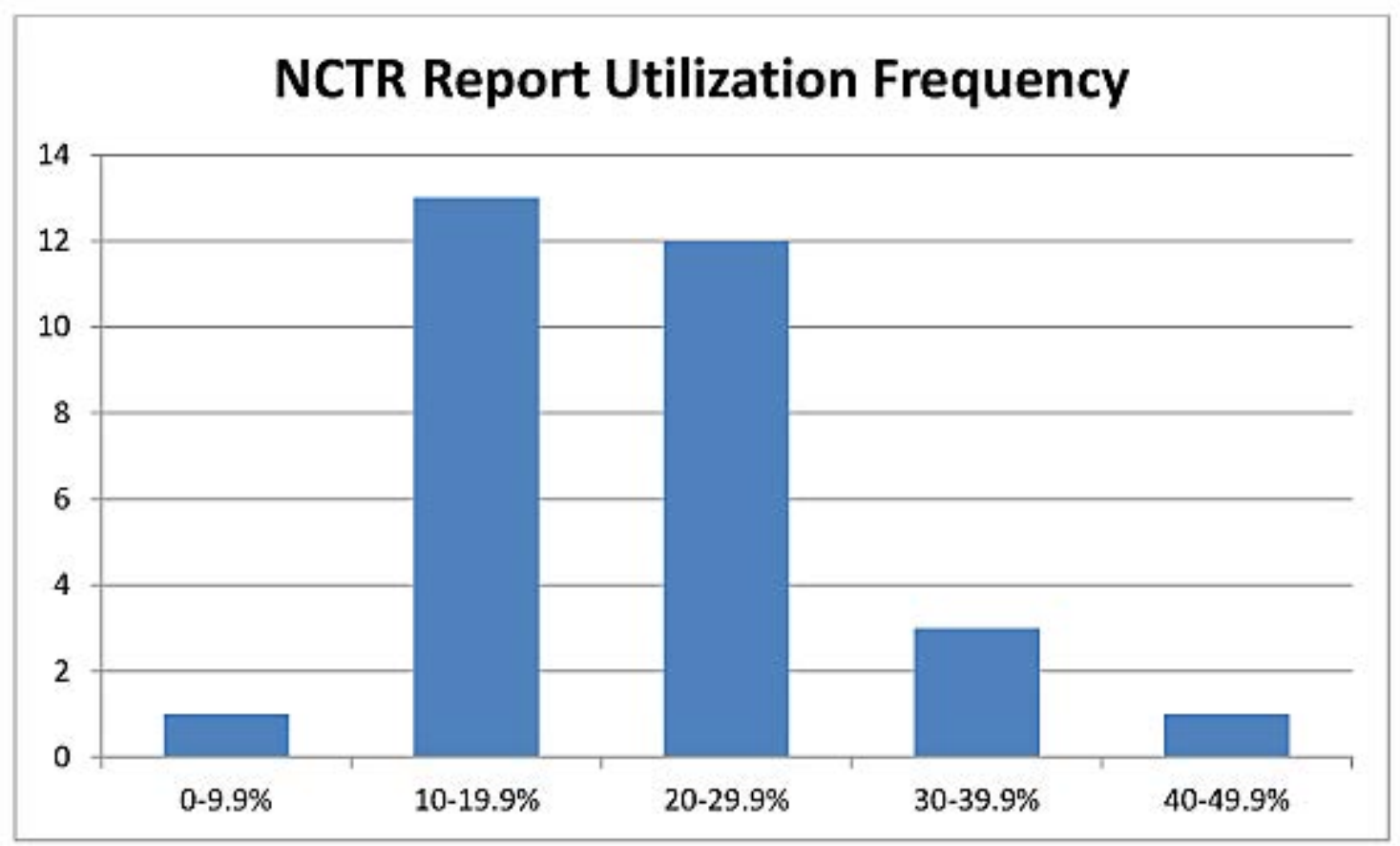

Figure 1: Report Utilization Frequency

Several respondents to the on line survey provided individual comments; these are included in Appendix B.

\section{NCTR Website Search Results}

Table 3 provides a summary of page views, unique page views, average time spent on page, and bounce rate for each project.

Table 3: NCTR Website Search Results

\begin{tabular}{|c|c|c|c|c|c|c|c|}
\hline & Title & Author & Project & $\begin{array}{l}\text { Page } \\
\text { Views }\end{array}$ & $\begin{array}{l}\text { Unique } \\
\text { Page } \\
\text { Views }\end{array}$ & $\begin{array}{l}\text { Average } \\
\text { Time On } \\
\text { Page }\end{array}$ & $\begin{array}{c}\text { Bounce } \\
\text { Rate }\end{array}$ \\
\hline 1 & $\begin{array}{l}\text { Smart Phone Application } \\
\text { to Influence Travel } \\
\text { Behavior (TRAC-IT } \\
\text { Phase 3) }\end{array}$ & Barbeau & BD549-35 & 1252 & 1076 & $1: 55$ & $76.01 \%$ \\
\hline 2 & $\begin{array}{l}\text { Guidebook on Using } \\
\text { American Community } \\
\text { Survey Data for Transit } \\
\text { Planning }\end{array}$ & Chu & $\begin{array}{c}\text { BDK85977- } \\
02\end{array}$ & 751 & 591 & $2: 04$ & $70.51 \%$ \\
\hline 3 & $\begin{array}{l}\text { Travel Assistance Device } \\
\text { (TAD) to Aid Transit } \\
\text { Riders with Special } \\
\text { Needs }\end{array}$ & Barbeau & BD549-33 & 726 & 621 & $2: 36$ & $77.89 \%$ \\
\hline
\end{tabular}


Table 3: NCTR Website Search Results (cont'd)

\begin{tabular}{|c|c|c|c|c|c|c|c|}
\hline & Title & Author & Project & $\begin{array}{l}\text { Page } \\
\text { Views }\end{array}$ & $\begin{array}{l}\text { Unique } \\
\text { Page } \\
\text { Views }\end{array}$ & $\begin{array}{l}\text { Average } \\
\text { Time On } \\
\text { Page }\end{array}$ & $\begin{array}{l}\text { Bounce } \\
\text { Rate }\end{array}$ \\
\hline 4 & $\begin{array}{l}\text { Best Practices In Transit } \\
\text { Services Planning }\end{array}$ & Goodwill & BD549-38 & 841 & 706 & $1: 29$ & $39.52 \%$ \\
\hline 5 & $\begin{array}{l}\text { Exploration of a Shift in } \\
\text { Household } \\
\text { Transportation Spending } \\
\text { from Vehicles to Public } \\
\text { Transportation }\end{array}$ & Polzin & BD549-43 & 1028 & 872 & $1: 26$ & $55.45 \%$ \\
\hline 6 & $\begin{array}{l}\text { Transit Ridership, } \\
\text { Reliability and Retention }\end{array}$ & Perk & BD549-32 & 225 & 195 & $0: 49$ & $18.18 \%$ \\
\hline 7 & $\begin{array}{l}\text { Transit Extraboard } \\
\text { Management-Optimum } \\
\text { Sizing and Strategies }\end{array}$ & DeAnnuntis & BD549-23 & 58 & 43 & $3: 02$ & $73.08 \%$ \\
\hline 8 & $\begin{array}{l}\text { Synthesis of research on } \\
\text { Value of Time and Value } \\
\text { of Reliability }\end{array}$ & Concas & BD549-46 & 392 & 326 & $0: 59$ & $26.36 \%$ \\
\hline 9 & $\begin{array}{l}\text { Development of } \\
\text { Comprehensive } \\
\text { Guidance on Obtaining } \\
\text { Service Consumed Data } \\
\text { for National Transit } \\
\text { Database (NTD) }\end{array}$ & Chu & BD549-47 & 379 & 347 & $1: 25$ & $47.83 \%$ \\
\hline 10 & $\begin{array}{l}\text { Evaluation of Smart } \\
\text { Video for Transit Event } \\
\text { Detection }\end{array}$ & Sapper & BD549-49 & 272 & 229 & $1: 45$ & $39.82 \%$ \\
\hline 11 & $\begin{array}{l}\text { Evaluation of Electronic } \\
\text { Data Recorder for } \\
\text { Incident Investigation, } \\
\text { Driver Performance and } \\
\text { Vehicle Maintenance }\end{array}$ & Sapper & BD549-50 & 242 & 213 & $1: 23$ & $51.82 \%$ \\
\hline 12 & $\begin{array}{l}\text { Quantifying Net Social } \\
\text { Benefits of Vehicle Trip } \\
\text { Reduction Impacts to } \\
\text { make Existing Road } \\
\text { Infrastructure Perform } \\
\text { Better-Guidance for } \\
\text { Customizing the } \\
\text { TRIMMS Model to Aid } \\
\text { Local, Regional and } \\
\text { State Decision Makers }\end{array}$ & Concas & BD549-52 & 1445 & 1203 & $1: 27$ & $39.10 \%$ \\
\hline 13 & $\begin{array}{l}\text { Utilizing Information } \\
\text { Technology in } \\
\text { Innovative Marketing } \\
\text { Approaches for Public } \\
\text { Transportation }\end{array}$ & Morris & BD549-53 & 606 & 511 & $1: 43$ & $49.43 \%$ \\
\hline
\end{tabular}


Table 3: NCTR Website Search Results (cont'd)

\begin{tabular}{|c|c|c|c|c|c|c|c|}
\hline & Title & Author & Project & $\begin{array}{l}\text { Page } \\
\text { Views }\end{array}$ & $\begin{array}{l}\text { Unique } \\
\text { Page } \\
\text { Views }\end{array}$ & $\begin{array}{l}\text { Average } \\
\text { Time On } \\
\text { Page }\end{array}$ & $\begin{array}{c}\text { Bounce } \\
\text { Rate }\end{array}$ \\
\hline 14 & $\begin{array}{l}\text { Testing the Impact of } \\
\text { Personalized Feedback } \\
\text { on Household Travel } \\
\text { Behavior }\end{array}$ & Winters & BD549-24 & 363 & 307 & $1: 36$ & $38.73 \%$ \\
\hline 15 & $\begin{array}{l}\text { Guidelines and } \\
\text { Performance Measures } \\
\text { to Incorporate Transit } \\
\text { and Other Multimodal } \\
\text { Considerations into the } \\
\text { FDOT DRI Review } \\
\text { Process }\end{array}$ & Seggerman & BD549-31 & 238 & 202 & $1: 12$ & $36.47 \%$ \\
\hline 16 & $\begin{array}{l}\text { Programs that Match } \\
\text { Seniors with Volunteer } \\
\text { Drivers }\end{array}$ & Hendricks & BD549-41 & 1370 & 1157 & $2: 00$ & $71.81 \%$ \\
\hline 17 & $\begin{array}{l}\text { Developing a Printed } \\
\text { Transit Information } \\
\text { Material Design Manual }\end{array}$ & Cain & BD549-29 & 2208 & 1781 & $2: 00$ & $61.78 \%$ \\
\hline 18 & $\begin{array}{l}\text { Guidebook for Start-up } \\
\text { Transit Agencies }\end{array}$ & Goodwill & BD549-14 & - & - & - & - \\
\hline 19 & $\begin{array}{l}\text { Developing a Technique } \\
\text { that Predicts the } \\
\text { Impacts of TDM on a } \\
\text { Transportation System }\end{array}$ & Georggi & $\begin{array}{l}\text { BDK85977- } \\
06\end{array}$ & 304 & 260 & $1: 54$ & $47.15 \%$ \\
\hline 20 & $\begin{array}{l}\text { Evaluation of Camera } \\
\text { Based Systems to } \\
\text { Reduce Transit Bus Side } \\
\text { Collisions }\end{array}$ & Lin & $\begin{array}{l}\text { BDK85977- } \\
\quad 08\end{array}$ & - & - & - & - \\
\hline 21 & $\begin{array}{l}\text { Investigation of the } \\
\text { Feasibility of Toll and } \\
\text { Transit Agency Equity } \\
\text { Sharing }\end{array}$ & Reich & $\begin{array}{l}\text { BDK85977- } \\
09\end{array}$ & 123 & 102 & $1: 34$ & $58.93 \%$ \\
\hline 22 & $\begin{array}{l}\text { Regional Fare Policy and } \\
\text { Fare Allocation, } \\
\text { Innovations in Fare } \\
\text { Equipment and Data } \\
\text { Collection }\end{array}$ & Joslin & BD549-51 & 248 & 185 & $1: 59$ & $71.15 \%$ \\
\hline 23 & $\begin{array}{l}\text { Creative Ways to } \\
\text { Manage Paratransit } \\
\text { Costs }\end{array}$ & Goodwill & BD549-28 & 333 & 265 & $1: 20$ & $46.46 \%$ \\
\hline 24 & $\begin{array}{l}\text { Integrating Transit and } \\
\text { Urban Form }\end{array}$ & Concas & BD549-37 & 542 & 449 & $2: 08$ & $31.93 \%$ \\
\hline 25 & $\begin{array}{l}\text { Development of Large } \\
\text { Bus/Small Bus Decision } \\
\text { Support Tool }\end{array}$ & Reich & BD549-39 & 1390 & 1136 & $1: 46$ & $38.69 \%$ \\
\hline
\end{tabular}


Table 3: NCTR Website Search Results (cont'd)

\begin{tabular}{|c|c|c|c|c|c|c|c|}
\hline & Title & Author & Project & $\begin{array}{l}\text { Page } \\
\text { Views }\end{array}$ & $\begin{array}{l}\text { Unique } \\
\text { Page } \\
\text { Views }\end{array}$ & $\begin{array}{l}\text { Average } \\
\text { Time On } \\
\text { Page }\end{array}$ & $\begin{array}{c}\text { Bounce } \\
\text { Rate }\end{array}$ \\
\hline 26 & $\begin{array}{l}\text { Development of NTD } \\
\text { Tool for Vanpool } \\
\text { Services }\end{array}$ & Chu & BD549-40 & 894 & 733 & $1: 27$ & $36.61 \%$ \\
\hline 27 & $\begin{array}{l}\text { Impacts of More } \\
\text { Rigorous ADA } \\
\text { Paratransit Eligibility } \\
\text { Assessments on Riders } \\
\text { with Disabilities }\end{array}$ & Sapper & BD549-44 & 572 & 491 & $1: 31$ & $33.99 \%$ \\
\hline 28 & $\begin{array}{l}\text { Enhancing Transit } \\
\text { Safety and Security with } \\
\text { Wireless Detection and } \\
\text { Communication } \\
\text { Technologies }\end{array}$ & Barbeau & BD549-45 & 281 & 232 & $2: 15$ & $58.82 \%$ \\
\hline 29 & $\begin{array}{l}\text { Toolbox for Transit } \\
\text { Event Investigation }\end{array}$ & Sapper & BD549-22 & 33 & 31 & $1: 12$ & $100 \%$ \\
\hline 30 & $\begin{array}{l}\text { Moving the Bus Back } \\
\text { Into Traffic Safety }\end{array}$ & Lin & BD549-34 & 24 & 21 & $1: 48$ & $84.21 \%$ \\
\hline
\end{tabular}

The number of page views ranged from 24 to 2,208 with an average of 612 page views. Figure 2 depicts the range of page view frequencies.

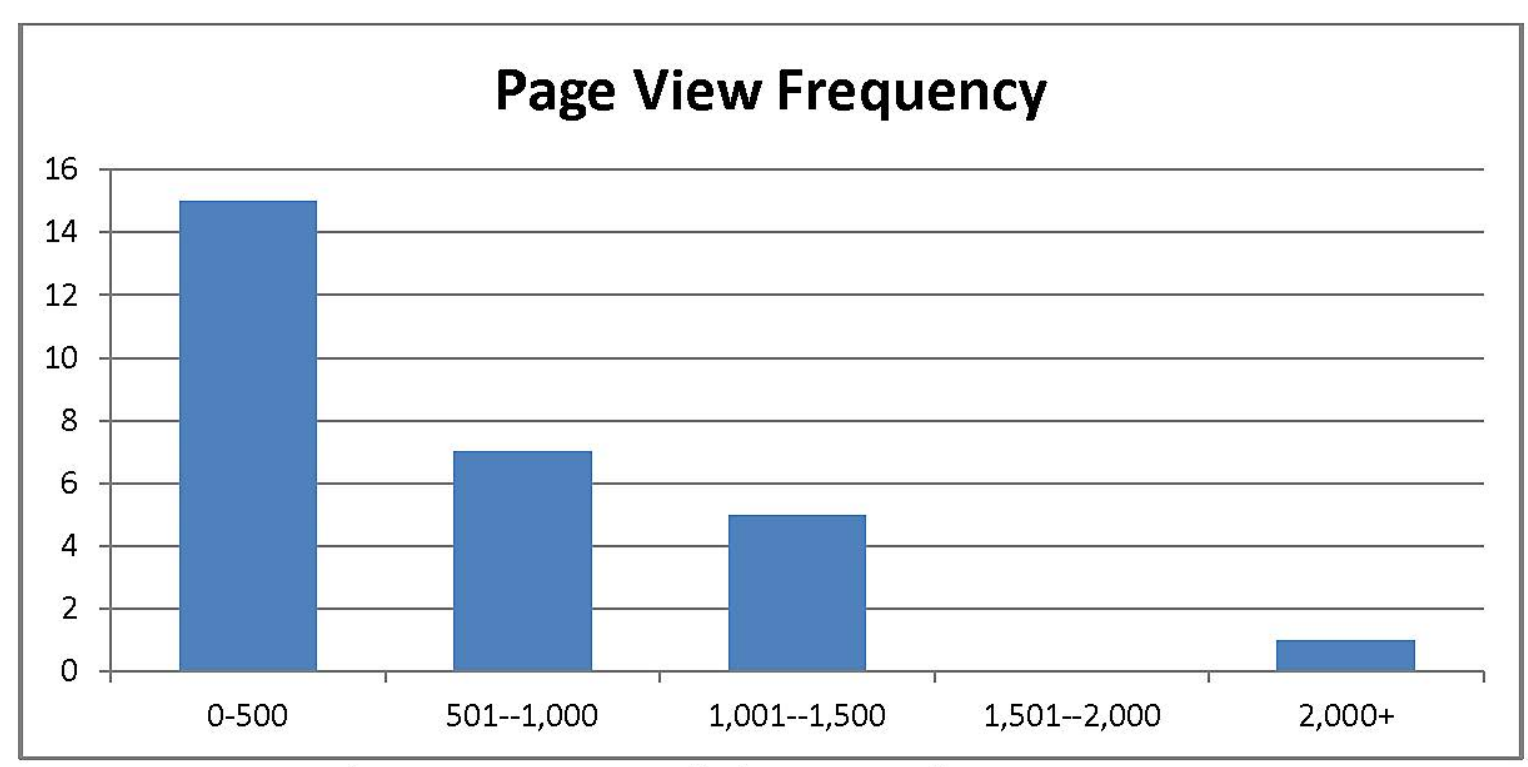

Figure 2: NCTR Website Page View Frequency 


\section{Quantitative Research Summary}

The quantitative data collected provide indirect indications of how well the 30 NCTR projects were utilized by professionals within the transportation industry. Projects that were cited more frequently in Google Scholar, viewed more frequently on the NCTR website, and that had a higher utilization rate reported through the on line survey were considered to have been more successful in reaching the transportation community. Table 4 provides a summary of the quantitative data collected.

Table 4: Quantitative Research Summary

\begin{tabular}{|c|c|c|c|c|c|c|}
\hline & Title & Author & Project & $\begin{array}{l}\text { Scholar } \\
\text { Search } \\
\text { Citations }\end{array}$ & $\begin{array}{l}\text { Web- } \\
\text { site } \\
\text { Page } \\
\text { Views }\end{array}$ & $\begin{array}{l}\text { Utilization } \\
\text { Rate-Listserv } \\
\text { Survey }\end{array}$ \\
\hline 1 & $\begin{array}{l}\text { Smart Phone Application to } \\
\text { Influence Travel Behavior } \\
\text { (TRAC-IT Phase 3) }\end{array}$ & Barbeau & BD549-35 & 8 & 1,252 & $26.6 \%$ \\
\hline 2 & $\begin{array}{l}\text { Guidebook on Using } \\
\text { American Community } \\
\text { Survey Data for Transit } \\
\text { Planning }\end{array}$ & Chu & $\begin{array}{c}\text { BDK85977 } \\
-02\end{array}$ & 8 & 751 & $23.2 \%$ \\
\hline 3 & $\begin{array}{l}\text { Travel Assistance Device } \\
\text { (TAD) to Aid Transit Riders } \\
\text { with Special Needs }\end{array}$ & Barbeau & BD549-33 & 5 & 726 & $26.9 \%$ \\
\hline 4 & $\begin{array}{l}\text { Best Practices In Transit } \\
\text { Services Planning }\end{array}$ & Goodwill & BD549-38 & 2 & 841 & $39.1 \%$ \\
\hline 5 & $\begin{array}{l}\text { Exploration of a Shift in } \\
\text { Household Transportation } \\
\text { Spending from Vehicles to } \\
\text { Public Transportation }\end{array}$ & Polzin & BD549-43 & 2 & 1,028 & $14.8 \%$ \\
\hline 6 & $\begin{array}{l}\text { Transit Ridership, Reliability } \\
\text { and Retention }\end{array}$ & Perk & BD549-32 & 3 & 58 & $12.8 \%$ \\
\hline 7 & $\begin{array}{l}\text { Transit Extraboard } \\
\text { Management-Optimum } \\
\text { Sizing and Strategies }\end{array}$ & DeAnnuntis & BD549-23 & 2 & 58 & $12.8 \%$ \\
\hline 8 & $\begin{array}{l}\text { Synthesis of Research on } \\
\text { Value of Time and Value of } \\
\text { Reliability }\end{array}$ & Concas & BD549-46 & 1 & 392 & $14.7 \%$ \\
\hline 9 & $\begin{array}{l}\text { Development of } \\
\text { Comprehensive Guidance on } \\
\text { Obtaining Service } \\
\text { Consumed Data for National } \\
\text { Transit Database (NTD) }\end{array}$ & Chu & BD549-47 & 0 & 894 & $14.5 \%$ \\
\hline 10 & $\begin{array}{l}\text { Evaluation of Smart Video } \\
\text { for Transit Event Detection }\end{array}$ & Sapper & BD549-49 & 1 & 272 & $11.1 \%$ \\
\hline
\end{tabular}


Table 4: Quantitative Research Summary (cont'd)

\begin{tabular}{|c|c|c|c|c|c|c|}
\hline & Title & Author & Project & $\begin{array}{l}\text { Scholar } \\
\text { Search } \\
\text { Citations }\end{array}$ & $\begin{array}{l}\text { Web- } \\
\text { site } \\
\text { Page } \\
\text { Views }\end{array}$ & $\begin{array}{l}\text { Utilization } \\
\text { Rate-Listserv } \\
\text { Survey }\end{array}$ \\
\hline 11 & $\begin{array}{l}\text { Evaluation of Electronic } \\
\text { Data Recorder for Incident } \\
\text { Investigation, Driver } \\
\text { Performance and Vehicle } \\
\text { Maintenance }\end{array}$ & Sapper & BD549-50 & 1 & 242 & $13.8 \%$ \\
\hline 12 & $\begin{array}{l}\text { Quantifying Net Social } \\
\text { Benefits of Vehicle Trip } \\
\text { Reduction Impacts to make } \\
\text { Existing Road Infrastructure } \\
\text { Perform Better-Guidance for } \\
\text { Customizing the TRIMMS } \\
\text { Model to Aid Local, Regional } \\
\text { and State Decision Makers }\end{array}$ & Concas & BD549-52 & 1 & 1,445 & $10.9 \%$ \\
\hline 13 & $\begin{array}{l}\text { Utilizing Information } \\
\text { Technology in Innovative } \\
\text { Marketing Approaches for } \\
\text { Public Transportation }\end{array}$ & Morris & BD549-53 & 1 & 606 & $19.1 \%$ \\
\hline 14 & $\begin{array}{l}\text { Testing the Impact of } \\
\text { Personalized Feedback on } \\
\text { Household Travel Behavior }\end{array}$ & Winters & BD549-24 & 1 & 333 & $22.3 \%$ \\
\hline 15 & $\begin{array}{l}\text { Guidelines and Performance } \\
\text { Measures to Incorporate } \\
\text { Transit and Other } \\
\text { Multimodal Considerations } \\
\text { into the FDOT DRI Review } \\
\text { Process }\end{array}$ & Seggerman & BD549-31 & 1 & 238 & $30.0 \%$ \\
\hline 16 & $\begin{array}{l}\text { Programs that Match } \\
\text { Seniors with Volunteer } \\
\text { Drivers }\end{array}$ & Hendricks & BD549-41 & 1 & 1,370 & $14.8 \%$ \\
\hline 17 & $\begin{array}{l}\text { Developing a Printed Transit } \\
\text { Information Material Design } \\
\text { Manual }\end{array}$ & Cain & BD549-29 & 1 & 2,208 & $21.8 \%$ \\
\hline 18 & $\begin{array}{l}\text { Guidebook for Start-up } \\
\text { Transit Agencies }\end{array}$ & Goodwill & BD549-14 & 0 & - & $14.9 \%$ \\
\hline 19 & $\begin{array}{l}\text { Developing a Technique that } \\
\text { Predicts the Impacts of TDM } \\
\text { on a Transportation System }\end{array}$ & Georggi & $\begin{array}{c}\text { BDK85977 } \\
-06\end{array}$ & 0 & 304 & $25.5 \%$ \\
\hline 20 & $\begin{array}{l}\text { Evaluation of Camera Based } \\
\text { Systems to Reduce Transit } \\
\text { Bus Side Collisions }\end{array}$ & Lin & $\begin{array}{c}\text { BDK85977 } \\
-08\end{array}$ & 0 & - & $20.9 \%$ \\
\hline 21 & $\begin{array}{l}\text { Investigation of the } \\
\text { Feasibility of Toll and Transit } \\
\text { Agency Equity Sharing }\end{array}$ & Reich & $\begin{array}{c}\text { BDK85977 } \\
-09\end{array}$ & 0 & 123 & $12.8 \%$ \\
\hline
\end{tabular}


Table 4: Quantitative Research Summary (cont'd)

\begin{tabular}{|c|c|c|c|c|c|c|}
\hline & Title & Author & Project & $\begin{array}{l}\text { Scholar } \\
\text { Search } \\
\text { Citations }\end{array}$ & $\begin{array}{l}\text { Web- } \\
\text { site } \\
\text { Page } \\
\text { Views }\end{array}$ & $\begin{array}{l}\text { Utilization } \\
\text { Rate-Listserv } \\
\text { Survey }\end{array}$ \\
\hline 22 & $\begin{array}{l}\text { Regional Fare Policy and } \\
\text { Fare Allocation, Innovations } \\
\text { in Fare Equipment and Data } \\
\text { Collection }\end{array}$ & Joslin & BD549-51 & 0 & 248 & $20.9 \%$ \\
\hline 23 & $\begin{array}{l}\text { Creative Ways to Manage } \\
\text { Paratransit Costs }\end{array}$ & Goodwill & BD549-28 & 0 & 333 & $22.3 \%$ \\
\hline 24 & $\begin{array}{l}\text { Integrating Transit and } \\
\text { Urban Form }\end{array}$ & Concas & BD549-37 & 0 & 542 & $27.1 \%$ \\
\hline 25 & $\begin{array}{l}\text { Development of Large } \\
\text { Bus/Small Bus Decision } \\
\text { Support Tool }\end{array}$ & Reich & BD549-39 & 0 & 1,390 & $21.8 \%$ \\
\hline 26 & $\begin{array}{l}\text { Development of NTD Tool } \\
\text { for Vanpool Services }\end{array}$ & Chu & BD549-40 & 1 & 379 & $9.2 \%$ \\
\hline 27 & $\begin{array}{l}\text { Impacts of More Rigorous } \\
\text { ADA Paratransit Eligibility } \\
\text { Assessments on Riders with } \\
\text { Disabilities }\end{array}$ & Sapper & BD549-44 & 0 & 572 & $23.1 \%$ \\
\hline 28 & $\begin{array}{l}\text { Enhancing Transit Safety } \\
\text { and Security with Wireless } \\
\text { Detection and } \\
\text { Communication } \\
\text { Technologies }\end{array}$ & Barbeau & BD549-45 & 0 & 281 & $17.4 \%$ \\
\hline 29 & $\begin{array}{l}\text { Toolbox for Transit Event } \\
\text { Investigation }\end{array}$ & Sapper & BD549-22 & 0 & 33 & $18.7 \%$ \\
\hline 30 & $\begin{array}{l}\text { Moving the Bus Back Into } \\
\text { Traffic Safety }\end{array}$ & Lin & BD549-34 & 0 & 24 & $30.6 \%$ \\
\hline
\end{tabular}

\section{Webcast Series}

CUTR/NCTR has established an online "Webcast Series" the purpose of which is to:

- Increase knowledge of transportation professionals and policy-makers in Florida and the balance of the nation by sharing the latest transportation research findings.

- Increase the reach of technology transfer, especially to those transportation professionals who are unable to travel to state and national conferences.

- Encourage discussion among participants and receive input on subjects requiring future research.

Since its inception through the date of this report, the findings of three of the NCTR projects analyzed in this report have been featured in the webcast series. Following is a summary of participant reactions to each webcast: 
- BD549-34: Moving the Bus Back Into Traffic Safely, Pei Sung Lin

- Over 65 percent of the webcast participants rated the webcast as very good or excellent.

- 50 percent of webcast participants indicated very good to excellent relevance to their current jobs.

- BDK85977-02: Guidebook on Using American Community Survey Data for Transit Planning, Xuehao Chu

- Over 70 percent of the webcast participants rated the webcast as very good or excellent.

- 67 percent of webcast participants indicated very good to excellent relevance to their current jobs

- BDK85977-08: Evaluation of Camera-Based Systems to Reduce Transit Bus Side Collisions, Pei Sung Lin

- Over 84 percent of the webcast participants rated the webcast as very good to excellent.

- 85 percent of webcast participants indicated very good to excellent relevance to their current jobs.

\section{Qualitative Research Findings}

The Principal Investigator for each of the 30 NCTR projects was contacted and personally interviewed to validate the status of his/her research project, identify the target customer base for the research product, identify research papers that had published, identify professional presentations that had been delivered, share feedback on the research from professional colleagues, and identify potential third-party stakeholders with whom the interviewer could speak.

For some projects, the project's Principal Investigator was able to identify individuals who were instrumental in providing guidance on the research, had utilized the research findings in some way, and/or who had helped share research findings with others. Where these third party stakeholders were identified, the author attempted to conduct follow-up interviews and gain additional insight into the outreach and technology transfer process. In many cases, the author's attempts to contact third party stakeholders proved unsuccessful.

Following is a summary of the qualitative research findings for each of the 30 projects. Interviewees are CUTR researchers unless otherwise noted.

\section{BD549-28: Creative Ways to Manage Paratransit Costs}

Interviewee: Jay Goodwill

The objectives of this project were to provide an overview of the different types of paratransit services offered throughout Florida and the United States; identify the trends in the costs of providing paratransit services; detail the major expense categories and factors 
impacting these cost centers; and identify public transportation providers who have been successful in developing and implementing cost containment strategies for controlling and reducing paratransit costs. The target customers for the research were public transit agencies, municipalities, counties, and others that provide or purchase paratransit services.

Outreach Summary: The Principal Investigator delivered a presentation on the subject at the 2009 FPTA/FDOT/CUTR Professional Development Workshop. ${ }^{1}$ The research was featured in the TRB E-Newsletter. ${ }^{2}$ A professional inquiry about the research was received from the Golden Gate Bridge, Highway and Transportation District. ${ }^{3}$

The research report has been added to the National RTAP library.

\section{BD549-32: Transit Ridership, Reliability and Retention}

Interviewee: Victoria Perk

The objective of this research was to explore three major components that affect transit ridership: travel time reliability, rider cessation, and the characteristics of infrequent riders. The project sought to determine the level of correlation between travel time reliability and transit ridership. The target customers for the research were public transit agencies.

Outreach Summary: The Principal Investigator presented research findings at the American Public Transportation Association's (APTA) Bus \& Paratransit Conference. ${ }^{4}$ The Principal Investigator received and responded to over a dozen email inquiries from participants at the APTA conferences.

\section{BD549-24: Testing the Impact of Personalized Feedback on Household Travel Behavior \\ Interviewees: Sean Barbeau and Nevine Georggi}

The objectives of this research were to "fine tune" the trial expert advice system prototype designed in Phase I by testing the system on a larger sample of households, expanding its capability to provide customized advice, and quantifying changes in travel behavior patterns after providing personalized travel advice to encourage individuals to choose a mix of travel choices to satisfy their travel needs rather than only choose the single occupant vehicle. The target customers for the research were households.

Outreach Summary: See discussion under NCTR Project BD549-35.

\section{BD549-34: Moving the Bus Back Into Traffic Safely}

Interviewees: Pei-Sung Lin and Aldo Fabregas (CUTR) and Paul Hughes (Velvac Incorporated)

The objectives of this research were to develop recommendations for MUTCD-compliant signage and pavement markings to address Yield to Bus safety issues; develop recommendations to the National Highway Traffic Safety Administration (NHTSA) on lighting configurations and/or signage for the back of transit buses that will be expected to reduce rear-end collisions; and develop recommendations for draft statutory language or 
modifications to existing statutes that would be needed to help increase viability of the Yield to Bus law. The targeted customers for this research were public transit agencies, state traffic engineers, highway safety and motor vehicle professionals, and the National Highway Traffic Safety Administration.

Outreach Summary: The deliverables from this project resulted in a request for a follow-up research initiative. According to Paul Hughes, Sales Director at Valvec, Dr. Lin's research is "opening up a whole new arena in safety consciousness" and Dr. Lin's work "will impact the entire United States." ${ }^{5}$ Additionally, a Denver Post article from January 2011 noted that findings from this project were the basis for equipment and process improvements made by several transit agencies in Colorado. ${ }^{6}$

\section{BD549-31: Performance Measures and Best Practices for Incorporating Transit into the FDOT DRI Review Process}

Interviewees: Karen Seggerman and Sara Hendricks

The objectives of this research were to identify and define performance measures to evaluate the consideration of transit in the FDOT DRI review process and how effectively FDOT staff reviews developments of regional impact regarding their impact to the state transportation system, particularly with consideration of transit. The target customers for this research were Florida DOT staff, Florida DOT district staff, local government planning departments, and the consultant community.

Outreach Summary: The research team published a transportation impact handbook in August 2010. ${ }^{7}$ The PI participated in a panel discussion of Developments of Regional Impacts at a Florida Public Transit Association annual meeting. ${ }^{8}$ The handbook is listed as a resource on FDOT's Transportation Impact Handbook website.

http://teachamerica.com/FDOT/TIH/TIH_061109.pdf

\section{BD549-51: Regional Fare Policy and Fare Allocation, Innovations in Fare Equipment and Data Collection}

Interviewee: Ann Joslin

The objectives of this research were to explore the experiences of transit systems across the United States that have implemented regionalized services and integrated fare systems to benefit those who may be considering such an arrangement including: identification of issues and concerns that transit agencies and financial institutions have encountered; customer and financial implications associated with various regional fare policy approaches; and documentation of fare-related technology issues and opportunities based on national experience. The target customers for this research were public transit agencies.

Outreach Summary: According to the Principal Investigator, Tri-Rail utilized the research findings and adapted its system to align with Miami-Dade Transit. The PI provided a copy of the final report to a Principal at Booz Allen Hamilton and the Information Specialist at the American Public Transportation Association and shared significant information with representatives of the Chicago Transit Authority. 


\section{BD549-22: Toolbox for Transit Event Investigation}

Interviewees: Deborah Sapper (CUTR) and Steve Dallman (Transportation Safety Institute)

The objectives of this research were to develop an event investigation procedural manual and a training module to assist transit agencies in implementing and complying with state requirements and identify best practices associated with bus transit accident and security incidents. The target customers for this research were public transit agencies and local/state governing bodies.

Outreach Summary: The PI fulfilled various inquiries from numerous transit agencies and organizations. Steve Dallman from TSI indicated the research findings had not yet been incorporated into their training curriculum.

\section{BD549-23: Transit Extraboard Management-Optimum Sizing and Strategies}

Interviewee: Chris DeAnnuntis

The objectives of this research were to summarize the process and develop a model application tool to aid small to mid-size transit agencies in managing their extraboard. The target customers for this research were small to mid-size transit agencies and state departments of transportation. The project's primary deliverable was a spreadsheet that provides transit agencies a tool to monitor inputs over time.

Outreach Summary: The Principal Investigator published a paper ${ }^{9}$ on the research and presented the research findings at a TRB Annual Meeting. ${ }^{10}$

\section{BD549-35: Smart Phone Application to Influence Travel Behavior (TRAC-IT Phase 3) \\ Interviewees: Sean Barbeau and Nevine Georggi}

The objectives of this research were to influence travel behavior by mode, route, or time of day through the integration of traveler information, GPS, location-aware services and TRACIT's PDA-based travel behavior advisory system into cell phone applications. The target customers for this research were data collection experts, travel behavior analysts, public transit users, public transit agencies, travel surveyors, and any traveler.

Outreach Summary: FDOT Projects BD549-24 and BD549-35 led to the creation of the Location-Aware Information Systems Laboratory (LAISL), a collaboration between CUTR and the Department of Computer Science and Engineering at the University of South Florida to improve quality of life by supporting the collection of data through GPSenabled cellular phones and wireless sensor networks, and transforming these data into meaningful information and actions. The Principal Investigators for FDOT Projects BD549-24 and BD549-35 have over a dozen patents pending. ${ }^{11}$ 


\section{BD549-29: Developing a Printed Transit Information Material Design Manual}

Interviewees: Alasdair Cain and Santiago Navaro (US DOT) and John Lancaster (Memphis Transit Authority)

The objective of this research was to develop a printed transit information material design manual capable of assisting transit agencies in the production of effective and consistent printed transit information materials. The primary aim of the study is to assist transit agencies in Florida, although it is recognized that the manual will also be a useful resource for transit agencies elsewhere. The target customers for this research were public transit systems and public transit customers.

Outreach Summary: The Principal Investigator co-authored a paper on the research findings which was published in the Transportation Research Record, ${ }^{12}$ which suggested cost savings of 26 percent and measurable ridership increases at the Fort Collins, Colorado transit system. The Principal Investigator presented research findings at over a dozen professional conferences across the United States. John Lancaster, Planning Manager at the Memphis Area Transit Authority, cited the research findings in his procurement of a professional firm to redesign the timetable and system maps for the Memphis Transit Authority. ${ }^{13}$

\section{BD549-33: Travel Assistance Device (TAD) to Aid Transit Riders with Special Needs}

Interviewees: Sean Barbeau and Nevine Georggi, (CUTR); Karen Wolf-Branigin (National Center for Senior Transportation) and Kevin Thigpen and Phil Cuffey (Dajuta)

The objectives of this research were to design and develop Travel Assistant Device prototype software for GPS-enabled cell phones that will guide transit riders with mental or cognitive disabilities in utilizing the transit system and create an online, map-based web page that will provide a caretaker and travel trainer with the means to remotely monitor the transit rider's location when desired. The target customers for this research were transit riders with cognitive disabilities, new transit riders, public transit travel trainers, transit agencies and tourists.

Outreach Summary: The Principal Investigator provided documentation of over 20 citations and references to the research including the Governor's Commission on Disabilities 2009 Report, ${ }^{14}$ Proceedings of the National Academy of Sciences' Transportation Research Board $90^{\text {th }}$ Annual Meeting ${ }^{15}$ (January 24, 2011), and the $37^{\text {th }}$ Association for Behavior Analysis International (ABAI) Annual Convention, Denver, CO (May 27-31, 2011). ${ }^{16}$

From a technology transfer and information exchange perspective, this project is the most successful of the 30 projects investigated by the author. Third-party stakeholder interviews revealed significant enthusiasm for and business interest in the research findings and the product generated by the research. The Hillsborough Area Transit Authority (HART) has incorporated the Traveler Assistance Device (TAD) as a key element of its travel training program. The Traveler Assistance Device has been licensed 
to Dajuta, a Tampa-based company who is spearheading the sale and distribution of the product and Dajuta representatives are very optimistic about their ability to sell and distribute the product to a large market. Karen Wolf Branigin, Director of the National Center on Senior Transportation, enthusiastically endorsed the TAD and expressed excitement about how TAD could improve access for people with disabilities. ${ }^{17}$

\section{BD549-39: Development of Large Bus/Small Bus Decision Support Tool}

Interviewee: Steve Reich

The objective of the research was to develop a decision support tool that could be used to assist transit agencies with vehicle deployment and acquisition choices. The target customers for this research were the Hillsborough Area Regional Transit (HART), LYNX, Jacksonville Transit Authority, PalmTran, Pinellas Suncoast Transit Authority, and all public transit agencies in Florida.

Outreach Summary: The research findings were provided to the Manager of Planning at the Memphis Transit Authority. ${ }^{18}$

\section{BD549-45: Enhancing Transit Safety and Security with Wireless Detection and Communication Technologies}

Interviewee: Sean Barbeau

The objectives of the research were to integrate remote Wireless Sensor Networks (WSNs) into existing two-way location-based multimedia communication systems for global positioning system (GPS)-enabled mobile phones developed by researchers at the University of South Florida (USF) and develop a prototype tool to serve as a pre-emptive mechanism to avoid potential disasters and be a catalyst for immediate response to mitigate the effects of an incident that has already occurred. The target customers for this research are public transit systems.

Outreach Summary: Sean J. Barbeau presented "Enhancing Transportation Safety and Security Through Wireless Detection and Communication Technology" at the 2009 Tanks \& Wireless Energy Technology ECO Conference, Tampa, on May 29, 2009. ${ }^{19}$

\section{BD549-40: Development of a NTD Tool for Vanpool Services}

Interviewee: Xuehao Chu

The objectives of this research were to develop a spreadsheet tool for agencies to sample, record, process, and report vanpool service and consumption data to the National Transit Database (NTD). The target customers for this research were Urbanized Area National Transit Data (NTD) Reporters with Vanpool Services. The research produced an Excel tool.

Outreach Summary: The research report was cited in FTA § 5307 Formula Earnings Potential from Vanpools in the DC Metropolitan Region for the Northern Virginia Transportation Commission, Revised: August 7, 2009. ${ }^{20}$ 


\section{BD549-37: Integrating Transit and Urban Form}

Interviewee: Sisinnio Concas

The objectives of this research were to develop an integrated approach to examining the relationships between transit design and urban form, indicating the relationship between transit and land-use variables, and synthesize academic research and practitioner-based work examining the relationships between transit design and urban land use. The target customers for this research were public transit and community planners.

Outreach Summary: This research project is designed to produce long-term benefits with limited or perhaps no immediate payoff. The findings provide a model for planners.

\section{BD549-41: Programs that Match Seniors with Volunteer Drivers}

Interviewee: Sara Hendricks

The objectives of this research were to identify and explore the challenges of developing and operating a volunteer senior mobility driving service, propose solutions to establish successful programs, and prepare guidance/best practices that could be used by a variety of audiences. The target customers for this research were public transit agencies, paratransit agencies, nonprofit organizations, and social service agencies that operate or plan to initiate volunteer transportation services.

Outreach Summary: The research team presented their findings at the 2011 Idaho Public Transit Association annual meeting, ${ }^{21}$ and at the 2010 Iowa Public Transit Association annual meeting. ${ }^{22}$ The Principal Investigator published an article in a 2009 issue of CUTRLines ${ }^{23}$ and presented research findings at the 2008 ACT International Conference Poster Session, ${ }^{24}$ the 2010 TRB Livable Communities conference, ${ }^{25}$ the 2011 TRB Annual Conference, ${ }^{26}$ and the 2011 ACT International Conference. ${ }^{27}$ The research findings have been incorporated into the Florida Commuter Choice Certificate Program, and the Principal Investigator's paper "Liability Issues of Volunteer Driving Programs" has been accepted for publication in Transportation Research Record. ${ }^{28}$

\section{BD549-38: Best Practices in Transit Services Planning}

Interviewees: Jay Goodwill and Ann Joslin

The objectives of this research were to identify existing best practices and develop a generic model approach that could be adapted and used by all Florida public transit agencies for fixed route bus transit service planning, specifically to include: Service Design Standards, Service Performance Measurements, and a standard Service Evaluation Methodology. The target customers for this research were fixed-route transit systems.

Outreach Summary: A professional inquiry was received from the New York City Transit Authority. The Principal Investigator delivered a presentation on the subject at a CUTR/FPTA Professional Development Workshop. ${ }^{29}$ 


\section{BD549-44: Impacts of More Rigorous ADA Paratransit Eligibility Assessments on Riders with Disabilities}

Interviewee: Deborah Sapper

The objective of the research was to study the impact of changes to the ADA complementary paratransit eligibility processes, with a specific focus on Florida public transit agencies. The target customers for this research were Florida's public transit agencies, transit riders who have disabilities and Florida's Developmental Disabilities Council. The research included telephone interviews with four Florida transit agencies including Broward County Transit, Jacksonville Transportation Authority, Regional Transit System in Gainesville, and Sarasota County Area Transit; and six transit agencies from outside the state including Metro Mobility in Minneapolis/St. Paul; The Lift in San Diego, California; GO! Bus in Grand Rapids, Michigan; Utah Transit Authority's Paratransit Service ADA Program in Salt Lake City and Trans-AID in Winston-Salem, North Carolina.

Outreach Summary: The findings were shared with each of the ten transit agencies referenced above that participated in the research. In addition, the Principal Investigators were interviewed by reporters from an online transportation publication based in New York City. ${ }^{30}$

\section{BD549-43: Exploration of a Shift in Household Transportation Spending from Vehicles to Public Transportation}

Interviewee: Steve Polzin

The objectives of the research were to explore several data sets to develop an understanding of the economic and travel implications that might arise were households to reduce auto ownership in response to better transit service in an urban area and provide information to support policy discussions that consider development of more transit intensive urban environments, with the expectation that these transit service investments can pay dividends in terms of lower household vehicle ownership and use costs.

This research project is unique from the perspective of the targeted customer. The research project is designed to serve as a contribution to the body of knowledge within the transportation industry and was not targeted to a specific customer market segment.

Outreach Summary: The Principal Investigator presented the report findings at the 2012 ACT Leadership Academy ${ }^{31}$ and recently published "The True Cost of Driving and Travel Behavior" in Planetizen magazine. ${ }^{32}$

\section{BD549-47: Development of Comprehensive Guidance on Obtaining Service Consumed Data for National Transit Database (NTD)}

Interviewee: Xuehao Chu

The objective of the research was to develop a new set of guidance to overcome the difficulties with the current FTA NTD guidance. The target customers for the research were existing and future NTD reporters. 
Outreach Summary: The Federal Transit Administration (FTA) conducted a webinar based on the research findings. ${ }^{33}$ FTA also published a manual based on the research and has posted a user's template on its website. ${ }^{34}$

\section{BDK85977-02: Guidebook on Using American Community Survey Data for Transit Planning}

Interviewee: Xuehao Chu

The objective of the research was to develop a tool that helps transportation planning professionals overcome difficulties in using ACS data. The target customers for the research were transportation planners.

Outreach Summary: The Principal Investigator presented the research findings through a webinar ${ }^{35}$ and has received inquiries from the Seattle Department of Transportation.

\section{BD549-52: Quantifying Net Social Benefits of Vehicle Trip Reduction Impacts to make Existing Road Infrastructure Perform Better-Guidance for Customizing the TRIMMS Model to Aid Local, Regional and State Decision Makers}

Interviewee: Sisinnio Concas

The objectives of the research were to obtain cost and benefit parameters to allow model customization at a regional level, and update and refine the model to allow the incorporation of regional parameters. The research also provided the documentation necessary to help professionals use the model by selecting the appropriate cost parameters, providing a reference to sources where such parameters can be obtained, and by offering general guidance on how to incorporate data already at their disposal.

Outreach Summary: This research project offers another excellent example of how NCTR research is producing products and tools that are adding value to the transportation industry. The primary deliverable from this research project was TRIMMS 2.0. The Principal Investigator provided documentation of over 12 citations and references to the research from both domestic and international sources including "Programs Using the TRIMMS Model;" ISATS2010: First International Symposium on Advances in Transport Sustainability, Arizona, November 17-19, 2010; ${ }^{36}$ "Estimating Net Social Benefits of Vehicle Trip Reductions with the TRIMMS Model," Transportation Research Board 89th Annual Meeting, Washington, D.C., January 10-14, 2010; ${ }^{37}$ "Estimating the Social Costs and Benefits of Transportation Demand Management Programs Using TRIMMS," TRB Integrated Corridor System Management Modeling-Best Practices Workshop, Irvine, CA, September 14-15, 2009; ${ }^{38}$ "Transportation Demand Management: The United States Experience," Technical Conferences on Mobility Management, Madrid, May 19-20, 2009; ${ }^{39}$ "Estimating Societal Benefits and Costs of Transportation Demand Management" Transportation Research Board $87^{\text {th }}$ Annual Meeting, Washington, D.C., January $13-17,2008 ;{ }^{40}$ and "The Demand for Vanpooling 
Services," Urban Transport XI-Urban Transport and the Environment in the 21st Century, Algarve, Portugal, April 12-14, 2005. ${ }^{41}$

\section{BD549-46: Synthesis of Research on Value of Time and Value of Reliability} Interviewee: Sisinnio Concas

The objectives of this research were to compile and synthesize current and past research on value of time (VOT) and the value of reliability of travel, compile extensive research into an application-oriented document that provides transportation modelers with reasonable ranges for VOT, and synthesize the more limited research on value of time reliability to provide practical guidance, based on current knowledge and to identify priority areas for further research. The target customers for this research were public transportation modelers.

Outreach Summary: The research findings were utilized by the U.S. Department of Transportation to help develop the Revised Departmental Guidance on Valuation of Travel Time in Economic Analysis ${ }^{42}$ and by the National Center for Freight and Infrastructure Research and Education in their work to incorporate toll-pricing policy into a micro-simulation model for long distance freight transportation. ${ }^{43}$

\section{BD549-49: Evaluation of Smart Video for Transit Event Detection}

Interviewee: Deborah Sapper

The objectives of this research were to study various commercial anomaly detection systems and develop an evaluation framework for commercial anomaly detection systems. The target customers for this research were public transit systems with video cameras and law enforcement agencies.

Outreach Summary: The Principal Investigator delivered presentations on the research findings at two separate CUTR/FPTA/FDOT Professional Development workshops. ${ }^{44}$

\section{BD549-50: Evaluation of Electronic Data Recorder for Incident Investigation, Driver Performance, and Vehicle Maintenance \\ Interviewee: Deborah Sapper}

The objectives of this research were to evaluate the benefits Event Data Recorders provide to transit agencies in three areas: incident investigation, driver performance, and vehicle maintenance. The target customers for this research were public transit agencies and administrators of Florida's Vehicle Procurement Program.

Outreach Summary: The Principal Investigator responded to a request for information from RITA. Research findings were posted on the Paul S. Sarbanes Transit in Parks Technical Assistance Center (TRIPTAC) Resource Center at the Western Transportation Institute (WTI) at Montana State University. 


\section{BDK85977-06: Developing a Technique that Predicts the Impacts of TDM on a Transportation System}

Interviewees: Nevine Georggi and Ed Hillsman

The objective of the research was to develop a technique that estimates the effect of implementing a mix of employer-based demand management strategies on the performance of a transportation system during peak commuting periods. The target market for the research was state, regional and metropolitan transportation planners.

Outreach Summary: According to the interviewees, the goals of the project were not achieved due principally to problems associated with data extraction. The final project report documented the research process and made recommendations for future research.

\section{BD549-53: Utilizing Information Technology in Innovative Marketing Approaches for Public Transportation}

Interviewees: William Morris (CUTR), "T" Harrison (Gainesville Transit Authority), and Jeremy Spinks (The Kidd Group)

The original objectives of the research were to identify innovative marketing techniques that have been attempted to date by, in, and surrounding the public transportation industry; solicit ideas for more unconventional applications that transit agencies and TDM professionals can consider; and provide tools for transit agencies to use the various sources for their own tailored marketing approaches. Early in the research process, the objective changed to create a guide to help transit agencies more effectively utilize information technology and social media. The target customers for the research were public transit agencies, TDM organizations, and transit marketing professionals.

Outreach Summary: The research produced "Routes to New Networks: A Guide to Social Media for the Public Transportation Industry." The Principal Investigator presented research findings at the 2009 meeting of the Florida Public Transit Association. ${ }^{45}$ The Gainesville Transit Authority has utilized the research findings to help guide its marketing efforts. ${ }^{46}$

\section{BDK85977-09: Investigation of the Feasibility of Toll and Transit Agency Equity Sharing}

Interviewees: Steve Reich and Martin Stone (Tampa Hillsborough Expressway Authority)

The objectives of the research were to frame the institutional constraints and opportunities for equity sharing that currently exist in the highway, transit, and toll agency realms and to identify statutory, regulatory, or policy changes that may be required. It will also lay out the pros and cons of the pursuit of bus toll lanes. The timing of the project's completion may allow for any constraints that are identified to be addressed in the upcoming multi-year federal transportation reauthorization. The target customers for this research were public transit agencies, toll agencies, expressway authorities, state departments of transportation, 
the Federal Highway Administration, the Federal Transit Administration, and private investors.

Outreach Summary: The Principal Investigator produced a white paper, which has proven "very helpful" (Stone) to the Tampa Hillsborough Expressway Authority. ${ }^{47}$ The findings of this research have led to additional funding to prove the concept to the Federal Highway Administration. The research findings may also serve to implement changes in national transportation funding policy.

\section{BDK85977-08: Evaluation of Camera Based Systems to Reduce Transit Bus Side Collisions}

Interviewees: Pei Sung Lin and Aldo Fabregas (CUTR) and Paul Hughes (Velvac

Incorporated)

The objective of this research was to evaluate the effectiveness of camera-based systems to reduce transit bus side crashes in a controlled environment. The target customers for this research were fixed route public transit agencies.

Outreach Summary: The Principal Investigator presented project findings via a CUTR/NCTR webinar. ${ }^{48}$ The research report was accepted for publication in Transportation Research Record. ${ }^{49}$ The Principal Investigator was quoted in the article "Eliminate the Blind Spot Once and for All," written by Mr. Paul Hughes, Specialty Vehicle Manager for Velvac, in BUSRide Magazine Online, http://busride.com/, October 15, 2011, BUSRide Magazine. ${ }^{50}$

\section{BD549-14: Guidebook for Start-up Transit Agencies}

Interviewees: Jay Goodwill and Ann Joslin

The objective of this research was to develop a guidebook for use by agencies in the process of initiating first time transit systems. The target customers for this research were communities and organizations looking to initiate public transit service, employees, and policy makers new to the public transit industry.

Outreach Summary: Ann Joslin communicated with and provided a PowerPoint presentation to representatives of North Carolina State University. ${ }^{51}$ She responded to requests and provided copies of the research report to representatives of the Santee Lynches Regional Council of Governments in Sumter, South Carolina and the American Public Transportation Association. ${ }^{52}$ She provided a copy of the research report to representatives of the Chicago RTA. The research report was posted on the Paul S. Sarbanes Transit in Parks Technical Assistance Center (TRIPTAC) Resource Center at the Western Transportation Institute (WTI) at Montana State University. Michael Davis, the transit manager for Atomic City Transit in Los Alamos, New Mexico, expressed gratitude "for the great work you did on the Guidebook for Startup Transit agencies." He said it was a really big help when they set up shop about three years ago. ${ }^{53}$ 


\section{Third-Party Stakeholder Interviews}

Section 3 discussed the results of the NCTR Listserv Survey. Four respondents to that survey offered personal contact information and were interviewed telephonically to gain additional insights into their utilization of NCTR research products and findings:

1. Professor Graham Currie from the University of Australia suggested the promotion of research findings could be enhanced by utilizing report titles that more clearly describe report contents. Professor Currie further suggested the NCTR website could be improved to make research documents easier to locate, download, and utilize.

2. John Hoffpauer is a recently retired member of the Little Rock MPO who currently offers consulting services. While aware of many NCTR products he had not utilized the findings from any project. He has participated in a number of NCTR webinars and concluded CUTR has great researchers who may not be great presenters.

3. Nick Sebastian from McElhanney Consulting appreciates the webinars. He discussed findings of Side Cameras on Buses (BDK85977-08) and Integrating Transit and Urban Form (B) with several of his clients, including BC Transit in Canada.

4. Wendy Silvani is the owner of Silvani Transportation Consulting. She indicated general satisfaction with NCTR products, but did not offer specific feedback to any research projects.

Additionally, the author interviewed two individuals with tangential connections to NCTR and the research conducted by the center. Glenn Wichard, Ph.D., is the Licensing Manager in the Division of Patents \& Licenses at the University of South Florida. Dr. Wichard offered to conduct seminars to help NCTR/CUTR researchers more fully understand how his office can help with technology transfer. Further, he recommended NCTR/CUTR researchers meet with him while developing scopes of work to help identify technology transfer options in advance of the research.

Harold "Skip" Paul is the Director of the Research Center at the Louisiana Department of Transportation. Mr. Paul discussed his dedication of a staff position entitled Implementation Engineer, who is responsible for technology transfer. Mr. Paul emphasized his personal involvement with bringing research results to practice; he ensures each project has a formal implementation strategy, each project has an engaged project review committee, and he evaluates each project manager's performance based on successful implementation.

\section{Supplemental Research}

The information-gathering instruments described in Chapter 3 were designed to assess knowledge of NCTR projects in general, without specific geographical or industry concentration. In analysis of the information, these instruments provided it was clear there remained an opportunity for an additional survey, targeting only high-level public transportation personnel in Florida. This information was desirable, as the projects had been 
scoped based on Florida public transportation needs and had been conducted by the University of South Florida and the Florida Department of Transportation.

Between June 22 and July 11, 2012, a five-question online survey was disseminated via personalized email to executive directors of Florida's 30 public transit agencies, each of Florida's 67 community transportation coordinators, 26 members of Florida's maintenance manager network, and 8 members of Florida's transit marketing network. In total, the survey was directed to 131 representatives of Florida's public transportation industry.

Consistent with the methodology discussed in Chapter 2, the survey was designed to help measure how well the findings and tools from 30 NCTR projects were known throughout Florida's public transit industry, assess how the findings and tools had been utilized, and identify specific outcomes from the utilization of these findings and tools.

The results of this task are discussed in detail in Appendix C. 


\section{Chapter 4 \\ Discussion}

\section{Validity of Hypotheses}

In general, the findings support the hypotheses that the results of NCTR research projects did reach the customer and that NCTR research findings did produce significant impacts throughout the transportation industry.

\section{Factors Affecting Results}

The major factor affecting the research results was data collection. In lieu of specific metrics that assessed how well research results reached the customer the quantitative data collected produced what could be considered indicators of utilization, such as website references and appearances in research and trade publications.

The qualitative data were difficult to obtain and susceptible to researcher bias. Principal Investigators were able to identify a limited number of customers who directly used the research results. When contacted, customers offered overwhelmingly positive comments about the NCTR research findings but provided limited examples of specific impacts of NCTR research.

\section{Implications}

The data collected and outlined in Chapters 2 and 3 offer solid evidence that NCTR-funded research projects have produced findings, outcomes, and tools that create direct benefit for transportation professionals, both domestically and internationally. The analysis of these data, particularly interviews with Principal Investigators and third-party stakeholders, also generated the following observation and implications.

\section{NCTR research leads to commercially viable products.}

- NCTR research produced the Travel Assistance Device (TAD), which has been licensed for sale and distribution to Dajuta, a private, for-profit firm based in Tampa, Florida.

- NCTR research produced a small bus/large bus decision tool, the second iteration of the TRIMMS model, and a data collection and analysis tool for FTA.

- NCTR researchers have published numerous guidebooks and manuals, such as the "Printed Transit Information Material Design Manual" and the "Guidebook for Startup Transit Agencies," many of which may have potential for commercial application.

NCTR research leads to patents.

United States Patent 8,036,679, "Optimizing Performance of Location-Aware Applications

Using State Machines" (www.uspto.gov), has been officially issued by the USPTO and 
directly originated (i.e., were discovered/developed during the research project) from the TRAC-IT 3 project (BD549-35).

The following patent applications were also generated as a direct result of this project:

- Adaptive Location Data Buffering for Location-Aware Applications

- System and Method for Determining Critical Points in LBS Applications

- System and Method for an Efficient General Architecture and Two-Layered Protocol in Support of Real-time Location-Aware Applications

- System and Method for Automatically Determining Purpose Information for Travel Behavior

Two patents that directly resulted from Travel Assistance Device (TAD) to Aid Transit Riders with Special Needs (BD549-33) have received a "Notice of Allowance" from the USPTO:

- Travel Assistance Device

- System and Method for Reliable Transit Stop Detection and Timely Rider Notification

Nine additional patent applications are indirectly related to both the TRACIT and TAD project, in that they were produced in subsequent research projects based on the output from the original TRACIT 1-3 and TAD projects.

\section{The University of South Florida has technology transfer resources.}

The Office of Patents and Licenses at the University of South Florida is available to assist CUTR's researchers better understand the technology transfer opportunities of their NCTRfunded projects. The advice and guidance afforded by the Office of Patents and Licenses is appropriate for all NCTR researchers.

\section{NCTR's technology transfer leaders.}

NCTR researchers who are most adept at technology transfer demonstrate a commitment to four specific tactics:

1. Aggressive pursuit of patents and licenses.

2. Willingness to take risks (and encouraging others to invest in our risk taking).

3. Commitment to wide dissemination from websites to Listservs to webinars to Twitter and other social media applications.

4. Integration of research results in training (not all CUTR teams have a training element).

\section{Administrative and institutional barriers inhibit technology transfer.}

Several institutional barriers hamper and constrain the ability of NCTR researchers to more actively engage in technology transfer. Despite limited financial resources to expend on technology transfer, limited time to engage in technology transfer activities, and minimal incentives to pursue technology transfer work, NCTR researchers have made significant strides in technology transfer and information sharing. More could be achieved if these institutional barriers were addressed. 
Awareness of research findings wanes within months of report publication. The quantitative data enumerated in Chapter 2 clearly make the point that the passage of time decreases the attention given to research findings posted on the NCTR website. The data reinforces the need for constant and continuous outreach efforts and the need to utilize a variety of communication channels to ensure research findings reach the target audiences in a timely manner.

NCTR's Principal Investigators vary in their understanding and commitment to ensuring research results are placed in the hands of targeted customers/users. Every Principal Investigator recognizes his/her role as a researcher; transportation research is the reason most joined CUTR. Conversely, not all researchers recognize their role and responsibility to technology transfer; these responsibilities are neither inherent in the "research" position nor are they outlined in any position description.

NCTR's Principal Investigators vary in their skill and experience to ensure research results reach the targeted customer.

Many Principal Investigators possess excellent transportation research skills and knowledge but lack the information exchange, communication, and marketing skill sets fundamental to ensuring the transfer of research findings to practical application.

NCTR can improve the ways research results are communicated to customers. NCTR/CUTR researchers tend to produce "typical" (i.e., lengthy) research reports and rely on traditional (e.g., papers and group presentations) to help communicate research findings to targeted customer groups, such as transportation providers or peer researchers.

Increased utilization of nontraditional communication channels (i.e., social media, webinars, one-to-one communication, and more "engaging" research reports) can enhance the utilization rate of NCTR-funded research.

NCTR research results are more likely to produce additional direct benefits if obtained and utilized by more customers.

The qualitative and quantitative data outlined in Chapters 2 and 3 offer strong evidence that NCTR-funded research projects have produced findings, outcomes, and tools that create direct benefit for transportation professionals, both domestically and internationally. The challenge for NCTR/CUTR is to maintain a high level of research while simultaneously improving outreach efforts.

NCTR research results impact the transportation industry.

While often anecdotal, many transportation professionals interviewed by the author expressed support for, appreciation of, and commendation for the outcomes of NCTR's research efforts.

NCTR projects are not always completed in a timely manner.

While NCTR's researchers demonstrate a commitment to schedule adherence, most projects researched by the author were not completed within the project schedule. Research is not a "production" activity with known/proven standards of how long an activity may take. 
Researchers often are doing things for the first time, which may translate into extended completions schedules.

NCTR problem statements clearly identify the target customer.

Each problem statement and scope of work reviewed by the author clearly identified the target audience and who could most benefit from the research findings. A clear focus on the target market facilitated the transfer of research findings and outcomes.

Not all NCTR-funded research would be candidates for technology transfer. The value of some NCTR-funded research is found in the additions made to the body of subject knowledge as well as the training provided to young, aspiring transportation professionals. Future decisions regarding NCTR-funded projects need to address this objective.

\section{Research products impact technology transfer success.}

NCTR research projects that produce tools or software for the transportation industry tend to achieve greater technology transfer success than research projects that produce guidelines or recommendations.

\section{Research objectives determine timing of impacts.}

Often, the primary outcome of a NCTR research project is the need for additional research, and the outcome of that additional research is further research. Therefore, measurable and transferable research results may not be realized until subsequent research projects are complete. However, those impacts may clearly be related to the initial research project, which may have been accomplished years prior. 


\section{Chapter 5 \\ Conclusions}

Overall, the data suggested NCTR research results reach the customer fairly well, customers utilized the results, and the results have a positive impact on the transportation industry. The impacts were a function of both the nature of the research and the degree to which the results were promoted throughout the industry.

The level of customer awareness-as measured by the number of project website hits, citations in professional journals, and survey responses-varied among the 30 subject projects. The level of customer awareness appeared to be a function of how adept and committed NCTR researchers were to two specific tactics:

1. Wide dissemination from websites to listservs to webinars to Twitter and other social media applications.

2. Integration of research results into training.

The data also suggested that the level of customer awareness was greater for more recently published reports and that passage of time decreased the degree of customer awareness.

\section{Summary of Conclusions}

During the course of the personal interviews, each Principal Investigator and each third party stakeholder was asked what thoughts, ideas, suggestions and/or process improvements they could offer that would improve NCTR's ability to ensure research project results reach the targeted audiences. Based on these inputs and insights from the author, a series of draft recommendations were developed and presented to the NCTR Program Director and members of the NCTR leadership team. Subsequently, the draft recommendations were presented to and discussed with an internal review committee consisting of three NCTR Principal Investigators.

Following are the issues that have been identified with corresponding proposed recommendations:

1. Issue: Most NCTR projects do not produce communicable results until the research project is complete. Additionally, NCTR projects have a well-defined project schedule that typically concludes with approval of the final project report. These two conditions create a situation where Principal Investigators have no timeframe within which to focus on publicizing and communication their research results.

Recommendation: Add a "research results outreach and dissemination" task to NCTR project schedules that commences upon final report approval. 
2. Issue: Principal Investigators vary in their understanding and embrace of their role and responsibility to ensure research results are placed in the hands of targeted customers/users. Concurrently, Principal Investigators vary in their skill and experience to ensure research results reach the targeted customer. As one PI stated "How do we fill the marketing vacuum that exists among Principal Investigators?"

Recommendation: Provide marketing and public outreach training to Principal Investigators.

3. Issue: Many Principal Investigators are unclear or unsure as to the role of NCTR's leadership team in managing and guiding the dissemination of research results.

Recommendation: NCTR's Management Team should assume a greater leadership role for ensuring research project results reach targeted audiences. NCTR's Management Team should meet annually with each Principal Investigator and specify their expectations for outreach and results dissemination.

4. Issue: During the course of an NCTR research project, Principal Investigators and FDOT Project Managers tend to focus on completing the research tasks and achieving the research objectives. Subsequently, "how do we ensure research results reach our target audiences?" is a question that tends not to be addressed until the final report is approved.

Recommendation: Include an element in each Quarterly Progress Report that specifies actions and planned activities the Principal Investigator AND the FDOT Project Manager has and will implement which focuses on outreach and results dissemination.

5. Issue: Many NCTR projects produce final reports which are considered too lengthy and, therefore, too time-consuming for many members of the target audiences to read.

Recommendation: Continue the current trend for Principal Investigators to utilize webinars as a means to share research results and incorporate social media as a tactic for sharing research results.

6. Issue: Many NCTR research initiatives tend to occur within a vacuum and subsequently many CUTR employees are unaware of NCTR research projects and the results those projects produce.

Recommendation: Better inform internal customers (CUTR employees) of the outcomes and products of NCTR projects.

7. Issue: Many NCTR research reports contain material that is appropriate for technology transfer via a training session or class and many Principal Investigators apply this tactic at FDOT, FPTA and CUTR-sponsored events throughout Florida. Lack of financial and temporal resources, combined with a natural "Florida orientation," often results in research results not being disseminated broadly throughout the country. 
Recommendation: Principal Investigators and the NCTR leadership team should identify and/or create training and education outside opportunities outside Florida.

8. Issue: Several Principal Investigators reflected that many NCTR research reports are posted in locations that are unknown and/or unused by transit practitioners and, subsequently, relevant research results remain hidden to those who could benefit most.

Recommendation: NCTR should identify and utilize "non- traditional" sources for publishing and posting NCTR research results and findings. It is recommended that PIs or their student assistants identify a list of professionals who will be the most likely to be interested, and invite those people to download a copy of the report and share it with those they know in the industry. APTA's electronic directory is one excellent source to identify the most likely users of NCTR research.

9. Issue: According to the Research and Innovative Technology Administration (RITA) "technology transfer ensures that research results become ideas, technologies or processes that contribute to the improvement of transportation." Further, RITA identifies "publication of research papers, presentations at conferences, training, field testing and deployment" as typical technology transfer activities.

Recommendation: Establish "technology transfer" mindset among CUTR researchers through ongoing education and training.

10. Issue: NCTR lacks both the marketing mindset, the business acumen and the professional expertise to bring research products to market. Principal Investigators are transportation professionals whose focus is on conducting applied research and who lack both the expertise and time to focus on tracking actions taken as a result of their completed research.

Recommendation: Retain a professional technology transfer specialist whose role is focused on technology transfer and working with PIs to bring research products to market.

11. Issue: The CUTR Career Path addresses the "typical" technology transfer activities-publications and presentations-and does not recognize or reward other technology transfer activities, such as patent and license applications.

Subsequently, there is some misalignment between the activities to which Principal Investigators allocate their time and the activities for which Principal Investigators are rewarded.

Recommendation: Revise the CUTR Career Path process to better incorporate technology transfer activities and successes.

12. Issue: Several Principal Investigators observed that NCTR projects provide significant professional development opportunities for students and younger researchers. 
Recommendation: Maximize the number of NCTR projects that involve a student and a task to allow PIs to help build the research capacity of students and younger researchers.

13. Issue: NCTR knows how many "hits" it receives on its website, but has no idea who or what agency might be looking at the research reports.

Recommendation: CUTR should consider ways to ask those who download reports to contact CUTR and let the researcher know how they are using the information from the report.

14. Issue: NCTR relies on traditional techniques for disseminating research results.

Recommendation: Diversify the technology transfer product offerings. Not everyone will request the full report or will utilize a webinar platform. Make sure all NCTR projects are listed in Research in Progress and FTA's Research Hub. 


\section{References}

${ }^{1}$ Florida Public Transit Association Professional Development Workshop. (2009). Tampa, FL

${ }^{2}$ Goodwill, J. (2009, March 31). Creative Ways to Manage Paratransit Costs. Tampa, FL, USA.

${ }^{3}$ Paulson, K. (2009, April 10). San Francisco , CA, USA.

${ }^{4}$ Perk, V. (2008). American Public Transportation Association, Bus \& Paratransit Conference. Factors that Influence Frequency of Transit use and Rider Retention. Austin, TX

${ }^{5}$ Hughes, P. (2011, June 10). Sales Director, Velvac. (M.-C. f. Audino, Interviewer)

${ }^{6}$ Leib, J. (2011, Jnauary 23). RTD buses getting rear-facing "yield" lights as part of new law. Denver Post.

7 Hendricks, S., and Seggerman, K. (2010). "Transportation Impact Handbook: Estimating the Transportation Impacts of Growth." Florida Department of Transportation.

8 34th Annual Florida Public Transit Association Conference. (2008). "Incorporating Transit and other Multimodal Strategies into the Florida Department of Transportation DRI Review Process." Sarasota: FDOT.

${ }^{9}$ DeAnnuntis, C., and Morris, W. (2008). "Transit Extraboard Management: Optimum Sizing and Strategies." Transportation Research Record 2072.

${ }^{10}$ DeAnnuntis, C., and Morris, W. (2008). Transportation Research Board Annual Meeting. Washington.

${ }^{11}$ Barbeau, S. (2012, Febraury 20).

12 Cain, A., and LaVelle, J. (2010). "Five Phase Approach to Improve Design of Printed Transit Information and to Monitor Impact of Customer Satisfaction." Transportation Research Record 2144.

13 Lancaster, J. (2011, July 27). Manager of Planning, Memphis Transit Authority; M. C. Audino, Interviewer.

14 Barbeau, S. (2009). Florida Governor's Commission on Disabilities, 2009 Report.

${ }^{15}$ Barbeau, S., and Georgii, N. (2011). Proceedings of the National Academy of Sciences' Transportation Research Board 90th Annual Meeting. Washington, DC.

${ }^{16}$ Barbeau, S., and Georggi, N. (2011). 37th Association for Behavior Analysis International (ABAI) Annual Convention. Denver.

17 Wolf-Branigin, K. (2011, September 2). Director, National Center for Senior Transportation; M. C. Audino, Interviewer.

${ }^{18}$ Audino, M. (2011, August 9). Faculty, Center for Urban Transportation Research. Tampa, $\mathrm{FL}$, USA.

${ }^{19}$ Barbeau, S. (2009). "Enhancing Transportation Safety and Security Through Wireless Detection and Communication Technology." Tanks and Wireless Energy Technology ECO Conference,Tampa.

${ }^{20}$ Chu, X. (2009). FTA 5307 Formula Earnings from Vanpools in the DC Metropolitan Region.

${ }^{21}$ Audino, M. (2011). "A Generosity-Based Solution to Idaho's Mobility Challenges." Community Transportation Association of Idaho.

22 Audino, M. (2010). "Volunteer Transportation." Iowa Public Transit Association Annual Meeting.

${ }^{23}$ Hendricks, S. (2009). Study Recommendations Support Volunteer Driving Programs for Seniors. CUTRLines 19(1), Center for Urban Transportation Research. 
${ }^{24}$ Hendricks, S. (2009). Association for Commuter Transportation International Conference, Chicago.

${ }^{25}$ Hendricks, S. (2010). Transportation for Livable Communitites, Washington, D.C.

${ }^{26}$ Hendricks, S. (2011). Transportation Research Board, Annual Meeting, Washington, D.C.

${ }^{27}$ Hendricks, S. (2011). Association for Commuter Transportation International Conference, Chicago.

${ }^{28}$ Audino, M., and Hendricks, S. (2012, March). "Livability Issues of Volunteer Driving Programs." Transportation Research Record.

${ }^{29}$ Goodwill, J., and Joslin, A. (2009). "Best Practices in Transit Services Planning." CUTR/FPTA Professional Development Workshop, Tampa.

${ }^{30}$ Sapper, D. (2009, May 6). Transportation Access Blog. Retrieved May 6, 2009, from Transportation Access: http://www.nyctransportationaccess.com/.

31 "The Future of Public Transportation," ACT Leadership Academy, April 16, 2012, Tampa.

32 "The True Cost of Driving and Travel Behavior," April 30, Planetizen, http://www.planetizen.com/node/56493.

${ }^{33}$ Chu, X. (2010, April). National Transit Database Sampling Requirements and Guidance Webinar.

${ }^{34}$ Chu, X. (2009). National Transit Database Sampling Manual. United States Department of Transportation (USDOT), Federal Transit Administration (FTA).

${ }^{35}$ Chu, X. (2011, March 24). The American Community Survey (ACS) Statistical Analyzer Webinar.

${ }^{36}$ Concas, S. (2010). "Programs Using the TRIMMS Model." ISATS2010, First International Symposium on Advances in Transport Sustainability, Arizona.

37 Concas, S. (2010). "Estimating Net Social Benefits of Vehicle Trip Reductions with the TRIMMS Model." Transportation Research Board 89th Annual Meeting, Washington, D.C.

${ }^{38}$ Concas, S. (2009)." Estimating the Social Costs and Benefits of Transportation Demand Management Programs Using TRIMMS." TRB Integrated Corridor System Management Modeling-Best Practices Workshop, Irvine.

39 Concas, S. (2009). "Transportation Demand Management: The United States Experience." Technical Conferences on Mobility Management, Madrid.

${ }^{40}$ Concas, S. (2008). "Estimating Societal Benefits and Costs of Transportation Demand Management." Transportation Research Board 87th Annual Meeting, Washington, D.C.

${ }^{41}$ Concas, S. (2005). "The Demand for Vanpooling Services." Urban Transport XI-Urban Transport and the Environment in the 21st Century, Algarve.

${ }^{42}$ Concas, S. (2011). Revised Departmental Guidance on Valuation of Travel Time in Economic Analysis. Office of the Secretary of the United States Department of Transportation.

43 Concas, S. (2011). "Incorporating Toll Pricing Policy into a Microsimulation Model for Long Distance Freight Transportation." National Center for Freight and Infrastructure Research and Education.

${ }^{44}$ Sapper, D. (2009). "Evaluation of Smart Video for Transit Event Detection." CUTR/FPTA Professional Development Workshop, Tampa. 
${ }^{45}$ Morris, W. (2009). "Routes to New Networks: A Guide to Social Media for the Public Transportation Industry." CUTR/FPTA Professional Development Workshop, Tampa.

${ }^{46}$ Harrison, T. (2011, August). Marketing and Comunications Specialist, Gainesville Transit Authority; M. C. Audino, Interviewer.

${ }^{47}$ Stone, M. (2011, August 15). Planning Director, Tampa Hillsborough Expressway Authority; M. C. Audino, Interviewer.

${ }^{48}$ Lin, P. S., and Fabregas, A. (2011, May 19). "Evaluation of Camera-Based Systems to Reduce Transit Bus Side Collisions." Webcast Series, Center for Urban Transportation Research, Tampa.

${ }^{49}$ Kourtellis, A., Lin, P. S., and Lee, C. (2012, March). "Evaluation of Video Camera System to Reduce Side Collisions of Transit Buses." Transportation Research Record.

${ }^{50}$ Hughes, P. (2011, October 15). "Eliminate the Blind Spot Once and for All." Retrieved October 2011, from BUSRide Magaizine Online, http://busride.com/.

51 Joslin, A. (2009, June 2). "Guidebook for Start-up Transit Agencies" North Carolina.

52 Joslin, A. (2007, January 19). "Guidebook for Start-up Transit Agencies," Orlando.

53 Joslin, A., and Goodwill, J. (2011, April 20). "Guidebook for Start-up Transit Agencies." 


\section{Appendix A: Examples of Google Searches}

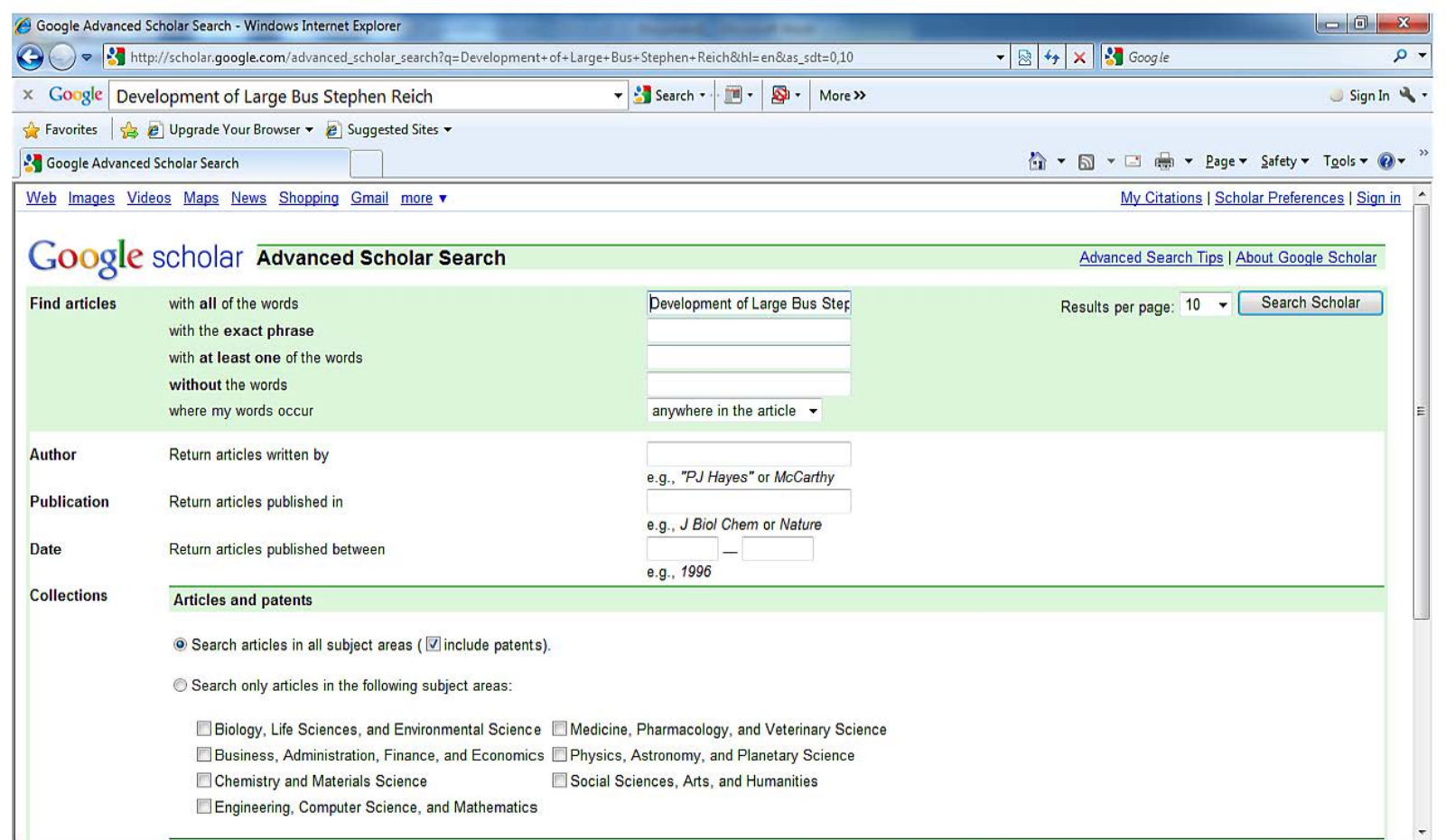

Figure A.1: Representative Screen Shot-Google Scholar Search

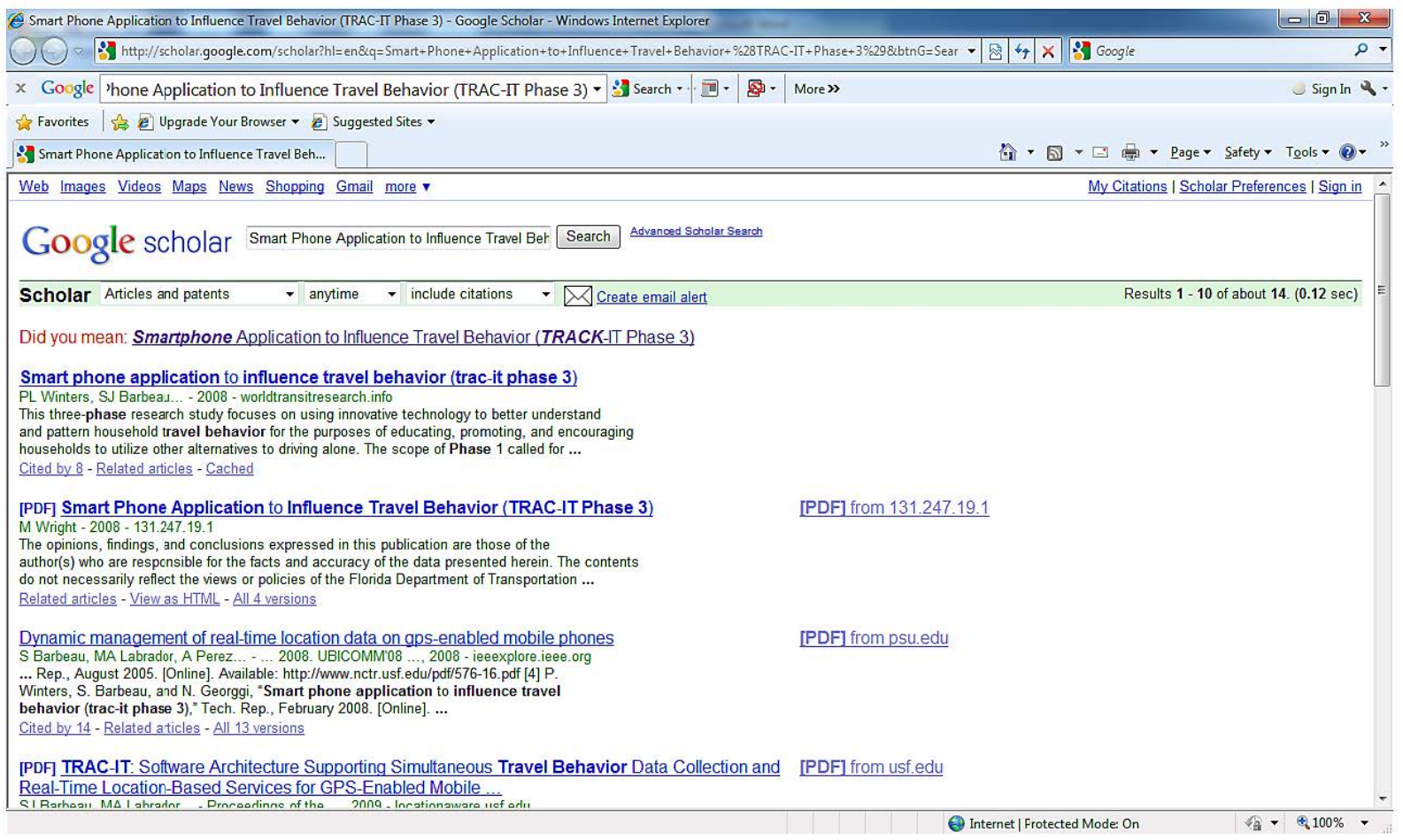

Figure A.2: Representative Search Results Page-Google Scholar 


\section{Appendix B: Listserv Survey Questions}

Question 1 provided a list of the 30 NCTR projects being evaluated and asked respondents to indicate if they or their organization utilized the findings or applied the tool of any of the projects on the list. Respondents were asked to reply "yes," "no," or "don't recall" to all projects on the list.

Question 2 asked respondents who answered "yes" to question 1 to describe how they used the results and/or tools.

Question 3 was an open-ended question which offered respondents the opportunity to suggest ways NCTR could improve the manner in which NCTR research results were distributed to the respondent or made public.

Question 4 provided respondents who answered "yes" to question 1 the opportunity to discuss their responses with the Principal Investigator by providing their name, email, and telephone number.

Responses to the open-ended question were not edited and are presented as originally submitted.

Table B.1: Responses to Open-Ended Question - Listserv Survey

\begin{tabular}{|c|c|c|}
\hline 1. & $\begin{array}{l}\text { The research studies cover various topics, including high technologies, } \\
\text { financial analysis, and maintenance cost evaluations projects. They are } \\
\text { very useful for enhancing a sustainable transportation environment. }\end{array}$ & $\begin{array}{r}\text { Wed, Aug 3, } 2011 \\
6: 12 \text { AM }\end{array}$ \\
\hline 2. & The current set up is okay with me. & $\begin{array}{r}\text { Fri, Jul 29, } 2011 \\
1: 26 \text { AM }\end{array}$ \\
\hline 3. & $\begin{array}{l}\text { I would not suggest improvements, the current manner of provision works } \\
\text { very well for us. }\end{array}$ & $\begin{array}{r}\text { Thu, Jul 28, } 2011 \\
2: 06 \text { PM }\end{array}$ \\
\hline 4. & $\begin{array}{l}\text { Some of the studies I was not aware of. I tend to check the CUTR website } \\
\text { and research specific information for questions or ways that may improve } \\
\text { our services. }\end{array}$ & $\begin{array}{r}\text { Wed, Jul 27, } 2011 \\
3: 53 \text { PM }\end{array}$ \\
\hline 5. & $\begin{array}{l}\text { Email us regarding the projects. Hold quarterly teleconference meetings } \\
\text { describing projects. Take input for new projects. Plan an open forum to } \\
\text { discuss national and local TDM issues. }\end{array}$ & $\begin{array}{r}\text { Tue, Jul 26, } 2011 \\
7: 49 \text { AM }\end{array}$ \\
\hline 6. & Like email and digital distribution. & $\begin{array}{r}\text { Mon, Jul 25, } 2011 \\
2: 14 \text { PM }\end{array}$ \\
\hline 7. & $\begin{array}{l}\text { By installing cameras on public transportation to observe how passengers } \\
\text { use the service and implement safer methods to avoid accidents etc. }\end{array}$ & $\begin{array}{r}\text { Mon, Jul 25, } 2011 \\
9: 39 \text { AM }\end{array}$ \\
\hline 8. & $\begin{array}{l}\text { Make the tools more clear and not just a random email announcement that } \\
\text { may be deleted. }\end{array}$ & $\begin{array}{r}\text { Mon, Jul 25, } 2011 \\
9: 26 \text { AM }\end{array}$ \\
\hline 9. & Continue to base all research on best practices statewide and nationwide. & $\begin{array}{r}\text { Mon, Jul 25, } 2011 \\
9: 15 \text { AM }\end{array}$ \\
\hline 10. & None. & $\begin{array}{r}\text { Mon, Jul 25, } 2011 \\
8: 10 \text { AM }\end{array}$ \\
\hline 11. & None ... great job CUTR staff! & $\begin{array}{r}\text { Mon, Jul 25, } 2011 \\
7: 57 \text { AM }\end{array}$ \\
\hline 12. & $\begin{array}{l}\text { I have no suggestions. I think the information is useful. For items that I } \\
\text { may have checked No or Don't Recall, I probably did not have a need for } \\
\text { that information at this time. }\end{array}$ & $\begin{array}{r}\text { Mon, Jul 25, } 2011 \\
7: 37 \text { AM }\end{array}$ \\
\hline 13. & $\begin{array}{l}\text { Quarterly publication of recent reports as a reminder of NCTR as a } \\
\text { resource. }\end{array}$ & $\begin{array}{r}\text { Mon, Jul 25, } 2011 \\
6: 25 \text { AM }\end{array}$ \\
\hline
\end{tabular}


Table B.1: Responses to Open-Ended Question - Listserv Survey (cont'd)

\begin{tabular}{|c|c|c|}
\hline 14. & $\begin{array}{l}\text { Research on topics that really matter to us operational folks - and not } \\
\text { necessarily academic topics. }\end{array}$ & $\begin{array}{r}\text { Mon, Jul 25, } 2011 \\
5: 47 \text { AM }\end{array}$ \\
\hline 15. & None & $\begin{array}{r}\text { Mon, Jul 25, } 2011 \\
5: 18 \text { AM }\end{array}$ \\
\hline 16. & Thorough training to all employees and public in general..., & $\begin{array}{r}\text { Sat, Jul 23, } 2011 \\
2: 42 \text { PM }\end{array}$ \\
\hline 17. & $\begin{array}{l}\text { I was not even aware of most of these reports and projects. I would } \\
\text { suggest that when they are published, you post them on the TRB weekly } \\
\text { site that gives short summaries of the latest research published with the } \\
\text { option for viewing the entire PDF reports. } \\
\text { This is also an opportunity for ACT to do something similar that simply lists } \\
\text { titles with links (like TRB does). The important thing is to send it weekly or } \\
\text { every other week, so people begin to look for it. } \\
\text { I am interested in reading several of these reports and wish I'd known } \\
\text { about them in the last few months. }\end{array}$ & $\begin{array}{r}\text { Fri, Jul 22, } 2011 \\
10: 39 \text { PM }\end{array}$ \\
\hline 18. & The present manner is fine for me. & $\begin{array}{r}\text { Fri, Jul 22, } 2011 \\
4: 33 \text { PM }\end{array}$ \\
\hline 19. & $\begin{array}{l}\text { No real improvements. The free webinars are wonderful and everything } \\
\text { seems to be easily available online which makes data retrieval a breeze. }\end{array}$ & $\begin{array}{r}\text { Fri, Jul 22, } 2011 \\
3: 24 \text { PM }\end{array}$ \\
\hline 20. & $\begin{array}{l}\text { 1. A Research Findings brief which specifically discusses } * \text { findings* for use } \\
\text { by practitioners, as opposed to more academic abstracts which simply } \\
\text { state that "findings were made." }\end{array}$ & $\begin{array}{r}\text { Fri, Jul 22, } 2011 \\
1: 40 \text { PM }\end{array}$ \\
\hline 21. & $\begin{array}{l}\text { Improve transit related issues via television when it affects routing and } \\
\text { scheduling. }\end{array}$ & $\begin{array}{r}\text { Fri, Jul 22, } 2011 \\
12: 27 \text { PM }\end{array}$ \\
\hline 22. & $\begin{array}{l}\text { Several titles were of interest but unknown to me. Your marketing of titles } \\
\text { can be proved. Your website could be proved. }\end{array}$ & $\begin{array}{r}\text { Fri, Jul 22, } 2011 \\
12: 23 \text { PM }\end{array}$ \\
\hline 23. & an email announcing the completion of the reports would be useful. & $\begin{array}{r}\text { Fri, Jul 22, } 2011 \\
11: 24 \text { AM }\end{array}$ \\
\hline 24. & $\begin{array}{l}\text { The transit studies have a wide scope of agency applications. Perhaps } \\
\text { developing a transit agency department representative list would help } \\
\text { specific divisions apply these studies when it's appropriate to their work. I } \\
\text { realize this isn't an easy task but it may help this valuable research } \\
\text { actually get applied in daily transit operations. }\end{array}$ & $\begin{array}{r}\text { Fri, Jul 22, } 2011 \\
11: 03 \text { AM }\end{array}$ \\
\hline 25. & $\mathrm{n} / \mathrm{a}$ & $\begin{array}{r}\text { Fri, Jul 22, } 2011 \\
10: 30 \text { AM }\end{array}$ \\
\hline 26. & None whatsoever. You're doing a terrific job of it. & $\begin{array}{r}\text { Fri, Jul 22, } 2011 \\
10: 21 \text { AM }\end{array}$ \\
\hline 27. & Maintain website links. & $\begin{array}{r}\text { Fri, Jul 22, } 2011 \\
10: 17 \text { AM }\end{array}$ \\
\hline 28. & $\begin{array}{l}\text { Get a decent editor and have the work reviewed by an editor before } \\
\text { coming to us. }\end{array}$ & $\begin{array}{r}\text { Fri, Jul 22, } 2011 \\
10: 14 \text { AM }\end{array}$ \\
\hline 29. & $\begin{array}{l}\text { I receive just reminders on the Thursday webinars. It might be helpful to } \\
\text { get an e-mail notice when you have a new publication. }\end{array}$ & $\begin{array}{r}\text { Fri, Jul 22, } 2011 \\
10: 11 \text { AM }\end{array}$ \\
\hline 30. & $\begin{array}{l}\text { Perhaps a briefer, lay-person summary (in addition to the summary } \\
\text { already provided). }\end{array}$ & $\begin{array}{r}\text { Fri, Jul 22, } 2011 \\
9: 05 \text { AM }\end{array}$ \\
\hline 31. & Email alerts when new reports come out. & $\begin{array}{r}\text { Fri, Jul 22, } 2011 \\
8: 21 \text { AM }\end{array}$ \\
\hline 32. & $\begin{array}{l}\text { More "concrete" examples/case studies. effect of hybrid/natural gas buses } \\
\text { on the environment. }\end{array}$ & $\begin{array}{r}\text { Fri, Jul 22, } 2011 \\
8: 08 \text { AM }\end{array}$ \\
\hline 33. & $\begin{array}{l}\text { TDM listserv, presentations at ACT national or regional conferences, even } \\
\text { posting this list on a quarterly basis.... }\end{array}$ & $\begin{array}{r}\text { Fri, Jul 22, } 2011 \\
8: 00 \text { AM }\end{array}$ \\
\hline
\end{tabular}


Table B.1: Responses to Open Ended Question - Listserv Survey (cont'd)

\begin{tabular}{|c|c|c|}
\hline 34. & $\begin{array}{l}\text { Make electronic copies available to us by sending email announcements of } \\
\text { recently completed projects that we can file and obtain when needed for } \\
\text { future efforts. }\end{array}$ & $\begin{array}{r}\text { Fri, Jul 22, } 2011 \\
7: 45 \text { AM }\end{array}$ \\
\hline 35. & None & $\begin{array}{r}\text { Fri, Jul 22, } 2011 \\
7: 23 \text { AM }\end{array}$ \\
\hline 36. & None to you - we simply need to remember to look and read. & $\begin{array}{r}\text { Fri, Jul 22, } 2011 \\
7: 19 \text { AM }\end{array}$ \\
\hline 37. & $\begin{array}{l}\text { I have just recently added this communication. I am unable to give you } \\
\text { feedback at this time. }\end{array}$ & $\begin{array}{r}\text { Fri, Jul 22, } 2011 \\
7: 05 \text { AM }\end{array}$ \\
\hline 38. & The e-blasts work well for me. & $\begin{array}{r}\text { Fri, Jul 22, } 2011 \\
6: 56 \text { AM }\end{array}$ \\
\hline 39. & $\begin{array}{l}\text { No specific suggestions. The material is generally well-researched and } \\
\text { well-prepared and has been very useful here. }\end{array}$ & $\begin{array}{r}\text { Fri, Jul 22, } 2011 \\
6: 33 \text { AM }\end{array}$ \\
\hline 40. & These should be sent out nationwide and seek endorsement from FTA. & $\begin{array}{r}\text { Fri, Jul 22, } 2011 \\
6: 32 \text { AM }\end{array}$ \\
\hline
\end{tabular}




\section{Appendix C: Supplemental Research}

\section{Introduction}

In this follow-up task to project BDK85 \#977-30, NCTR focused on assessing the outcomes and impacts of the results of 30 FDOT-sponsored NCTR research projects within Florida's public transportation industry. The goal of this task was to answer three primary research questions:

4. How well did the research results reach the customer?

5. How did the customer utilize the research results?

6. How did the research results impact the customer?

The task assessed the level of customer awareness of each research project and attempted to identify specific impacts of each research project. The task involved collection of both qualitative and quantitative data.

\section{Methodology}

Between June 22 and July 11, 2012, a five-question, online survey was disseminated via email to executive directors of Florida's 30 public transit agencies, each of Florida's 67 community transportation coordinators, 26 members of Florida's maintenance manager network, and 8 members of Florida's transit marketing network. In total, the survey was directed to 131 representatives of Florida's public transportation industry.

The survey was designed to help measure how well the findings and tools from 30 NCTR projects were known throughout Florida's public transit industry, assess how the findings and tools had been utilized, and identify specific outcomes from the utilization of these findings and tools.

\section{Findings}

A total of 18 survey responses were received. The survey response rate was $13.74 \%$. While 18 responses were received, not all respondents answered every question.

Question 1 provided a listing of the 30 NCTR projects and afforded respondents the opportunity to indicate-for each project-if they or their organization had utilized the findings or applied the tool from the research. Respondents could answer "yes," "no," or "don't know."

The percentage of respondents that indicated they had used NCTR research results ranged from 0 percent (Quantifying Net Social Benefits of Vehicle Trip Reduction Impacts to make Existing Road Infrastructure Perform Better-Guidance for Customizing the TRIMMS Model to Aid Local, Regional 
and State Decision Makers ) to 38.9 percent (Project BD549-38, Best Practices in Transit Services Planning).

A "yes" answer to Question 1 is assumed to imply that the respondent is both aware of the research report and has utilized the report. Subsequently, the average utilization rate was 17.24 percent. Table C.1 provides a summary of the responses to Question 1 of the survey.

Table C.1: Email Survey Summary Results

\begin{tabular}{|c|c|c|c|c|c|c|c|}
\hline & Title & Author & Project & Yes & No & $\begin{array}{l}\text { Don't } \\
\text { Recall }\end{array}$ & $\begin{array}{l}\text { Response } \\
\text { Count }\end{array}$ \\
\hline 1 & $\begin{array}{l}\text { Smart Phone Application } \\
\text { to Influence Travel } \\
\text { Behavior (TRAC-IT } \\
\text { Phase 3) }\end{array}$ & Barbeau & BD549-35 & $\begin{array}{c}3 \\
(17.6 \%)\end{array}$ & $\begin{array}{c}12 \\
(70.6 \%)\end{array}$ & $\begin{array}{c}2 \\
(11.8 \%)\end{array}$ & 17 \\
\hline 2 & $\begin{array}{l}\text { Guidebook on Using } \\
\text { American Community } \\
\text { Survey Data for Transit } \\
\text { Planning }\end{array}$ & Chu & $\begin{array}{l}\text { BDK85977- } \\
02\end{array}$ & $\begin{array}{c}2 \\
(11.1 \%)\end{array}$ & $\begin{array}{c}12 \\
(66.7 \%)\end{array}$ & $\begin{array}{c}4 \\
(22.8 \%)\end{array}$ & 18 \\
\hline 3 & $\begin{array}{l}\text { Travel Assistance Device } \\
\text { (TAD) to Aid Transit } \\
\text { Riders with Special } \\
\text { Needs }\end{array}$ & Barbeau & BD549-33 & $\begin{array}{c}2 \\
(11.8 \%)\end{array}$ & $\begin{array}{c}11 \\
(64.7 \%)\end{array}$ & $\begin{array}{c}4 \\
(23.5 \%)\end{array}$ & 17 \\
\hline 4 & $\begin{array}{l}\text { Best Practices In Transit } \\
\text { Services Planning }\end{array}$ & Goodwill & BD549-38 & $\begin{array}{c}7 \\
(38.9 \%)\end{array}$ & $\begin{array}{c}7 \\
(38.9 \%)\end{array}$ & $\begin{array}{c}4 \\
(22.2 \%)\end{array}$ & 18 \\
\hline 5 & $\begin{array}{l}\text { Exploration of a Shift in } \\
\text { Household } \\
\text { Transportation Spending } \\
\text { from Vehicles to Public } \\
\text { Transportation }\end{array}$ & Polzin & BD549-43 & $\begin{array}{c}2 \\
(11.1 \%)\end{array}$ & $\begin{array}{c}13 \\
(72.2 \%)\end{array}$ & $\begin{array}{c}3 \\
(16.7 \%)\end{array}$ & 18 \\
\hline 6 & $\begin{array}{l}\text { Transit Ridership, } \\
\text { Reliability and Retention }\end{array}$ & Perk & BD549-32 & $\begin{array}{c}6 \\
(35.3 \%)\end{array}$ & $\begin{array}{c}9 \\
(52.9 \%)\end{array}$ & $\begin{array}{c}2 \\
(11.8 \%)\end{array}$ & 17 \\
\hline 7 & $\begin{array}{l}\text { Transit Extraboard } \\
\text { Management-Optimum } \\
\text { Sizing and Strategies }\end{array}$ & DeAnnuntis & BD549-23 & $\begin{array}{c}3 \\
(17.6 \%)\end{array}$ & $\begin{array}{c}11 \\
(64.7 \%)\end{array}$ & $\begin{array}{c}3 \\
(17.6 \%)\end{array}$ & 17 \\
\hline 8 & $\begin{array}{l}\text { Synthesis of Research } \\
\text { on Value of Time and } \\
\text { Value of Reliability }\end{array}$ & Concas & BD549-46 & $\begin{array}{c}16 \\
(14.7 \%)\end{array}$ & $\begin{array}{c}77 \\
(70.6 \%)\end{array}$ & $\begin{array}{c}16 \\
(14.7 \%)\end{array}$ & 109 \\
\hline 9 & $\begin{array}{l}\text { Development of } \\
\text { Comprehensive } \\
\text { Guidance on Obtaining } \\
\text { Service Consumed Data } \\
\text { for National Transit } \\
\text { Database (NTD) }\end{array}$ & Chu & BD549-47 & $\begin{array}{c}10 \\
(9.2 \%)\end{array}$ & $\begin{array}{c}81 \\
(74.3 \%)\end{array}$ & $\begin{array}{c}18 \\
(16.5 \%)\end{array}$ & 109 \\
\hline 10 & $\begin{array}{l}\text { Evaluation of Smart } \\
\text { Video for Transit Event } \\
\text { Detection }\end{array}$ & Sapper & BD549-49 & $\begin{array}{c}12 \\
(11.1 \%)\end{array}$ & $\begin{array}{c}79 \\
(73.1 \%)\end{array}$ & $\begin{array}{c}17 \\
(15.7 \%)\end{array}$ & 108 \\
\hline
\end{tabular}


Table C.2: Email Survey Summary Results (cont'd)

\begin{tabular}{|c|c|c|c|c|c|c|c|}
\hline & Title & Author & Project & Yes & No & $\begin{array}{l}\text { Don't } \\
\text { Recall }\end{array}$ & $\begin{array}{l}\text { Response } \\
\text { Count }\end{array}$ \\
\hline 11 & $\begin{array}{l}\text { Evaluation of Electronic } \\
\text { Data Recorder for } \\
\text { Incident Investigation, } \\
\text { Driver Performance and } \\
\text { Vehicle Maintenance }\end{array}$ & Sapper & BD549-50 & $\begin{array}{c}15 \\
(13.8 \%)\end{array}$ & $\begin{array}{c}79 \\
(72.5 \%)\end{array}$ & $\begin{array}{c}15 \\
(13.8 \%)\end{array}$ & 109 \\
\hline 12 & $\begin{array}{l}\text { Quantifying Net Social } \\
\text { Benefits of Vehicle Trip } \\
\text { Reduction Impacts to } \\
\text { make Existing Road } \\
\text { Infrastructure Perform } \\
\text { Better-Guidance for } \\
\text { Customizing the } \\
\text { TRIMMS Model to Aid } \\
\text { Local, Regional and } \\
\text { State Decision Makers }\end{array}$ & Concas & BD549-52 & $\begin{array}{c}12 \\
(10.9 \%)\end{array}$ & $\begin{array}{c}78 \\
(70.9 \%)\end{array}$ & $\begin{array}{c}20 \\
(18.2 \%)\end{array}$ & 110 \\
\hline 13 & $\begin{array}{l}\text { Utilizing Information } \\
\text { Technology in } \\
\text { Innovative Marketing } \\
\text { Approaches for Public } \\
\text { Transportation }\end{array}$ & Morris & BD549-53 & $\begin{array}{c}21 \\
(19.1 \%)\end{array}$ & $\begin{array}{c}70 \\
(63.6 \%)\end{array}$ & $\begin{array}{c}19 \\
(17.3 \%)\end{array}$ & 109 \\
\hline 14 & $\begin{array}{l}\text { Testing the Impact of } \\
\text { Personalized Feedback } \\
\text { on Household Travel } \\
\text { Behavior }\end{array}$ & Winters & BD549-24 & $\begin{array}{c}17 \\
(15.5 \%)\end{array}$ & $\begin{array}{c}76 \\
(69.1 \%)\end{array}$ & $\begin{array}{c}17 \\
(15.5 \%)\end{array}$ & 110 \\
\hline 15 & $\begin{array}{l}\text { Guidelines and } \\
\text { Performance Measures } \\
\text { to Incorporate Transit } \\
\text { and Other Multimodal } \\
\text { Considerations into the } \\
\text { FDOT DRI Review } \\
\text { Process }\end{array}$ & Seggerman & BD549-31 & $\begin{array}{c}33 \\
(30.0 \%)\end{array}$ & $\begin{array}{c}63 \\
(57.3 \%)\end{array}$ & $\begin{array}{c}14 \\
(12.7 \%)\end{array}$ & 110 \\
\hline 16 & $\begin{array}{l}\text { Programs that Match } \\
\text { Seniors with Volunteer } \\
\text { Drivers }\end{array}$ & Hendricks & BD549-41 & $\begin{array}{c}16 \\
(14.8 \%)\end{array}$ & $\begin{array}{c}80 \\
(74.1 \%)\end{array}$ & $\begin{array}{c}12 \\
(11.1 \%)\end{array}$ & 108 \\
\hline 17 & $\begin{array}{l}\text { Developing a Printed } \\
\text { Transit Information } \\
\text { Material Design Manual }\end{array}$ & Cain & BD549-29 & $\begin{array}{c}24 \\
(21.8 \%)\end{array}$ & $\begin{array}{c}68 \\
(61.8 \%)\end{array}$ & $\begin{array}{c}18 \\
(16.4 \%)\end{array}$ & 110 \\
\hline 18 & $\begin{array}{l}\text { Guidebook for Start-up } \\
\text { Transit Agencies }\end{array}$ & Goodwill & BD549-14 & $\begin{array}{c}15 \\
(14.0 \%)\end{array}$ & $\begin{array}{c}76 \\
(71.0 \%)\end{array}$ & $\begin{array}{c}16 \\
(15.0 \%)\end{array}$ & 107 \\
\hline 19 & $\begin{array}{l}\text { Developing a Technique } \\
\text { that Predicts the } \\
\text { Impacts of TDM on a } \\
\text { Transportation System }\end{array}$ & Georggi & $\begin{array}{l}\text { BDK85977- } \\
06\end{array}$ & $\begin{array}{c}1 \\
(5.9 \%)\end{array}$ & $\begin{array}{c}14 \\
(82.4 \%)\end{array}$ & $\begin{array}{c}2 \\
(11.8 \%)\end{array}$ & 17 \\
\hline
\end{tabular}


Table C.3: Email Survey Summary Results (cont'd)

\begin{tabular}{|c|c|c|c|c|c|c|c|}
\hline & Title & Author & Project & Yes & No & $\begin{array}{l}\text { Don't } \\
\text { Recall }\end{array}$ & $\begin{array}{l}\text { Response } \\
\text { Count }\end{array}$ \\
\hline 20 & $\begin{array}{l}\text { Evaluation of Camera } \\
\text { Based Systems to } \\
\text { Reduce Transit Bus Side } \\
\text { Collisions }\end{array}$ & Lin & $\begin{array}{l}\text { BDK85977- } \\
08\end{array}$ & $\begin{array}{c}4 \\
(23.5 \%)\end{array}$ & $\begin{array}{c}10 \\
(58.8 \%)\end{array}$ & $\begin{array}{c}3 \\
(17.6 \%)\end{array}$ & 17 \\
\hline 21 & $\begin{array}{l}\text { Investigation of the } \\
\text { Feasibility of Toll and } \\
\text { Transit Agency Equity } \\
\text { Sharing }\end{array}$ & Reich & $\begin{array}{l}\text { BDK85977- } \\
09\end{array}$ & $\begin{array}{c}1 \\
(5.9 \%)\end{array}$ & $\begin{array}{c}12 \\
(70.6 \%)\end{array}$ & $\begin{array}{c}4 \\
(23.5 \%)\end{array}$ & 17 \\
\hline 22 & $\begin{array}{l}\text { Regional Fare Policy and } \\
\text { Fare Allocation, } \\
\text { Innovations in Fare } \\
\text { Equipment and Data } \\
\text { Collection }\end{array}$ & Joslin & BD549-51 & $\begin{array}{c}4 \\
(23.5 \%)\end{array}$ & $\begin{array}{c}10 \\
(58.8 \%)\end{array}$ & $\begin{array}{c}3 \\
(17.6 \%)\end{array}$ & 17 \\
\hline 23 & $\begin{array}{l}\text { Creative Ways to } \\
\text { Manage Paratransit } \\
\text { Costs }\end{array}$ & Goodwill & BD549-28 & $\begin{array}{c}3 \\
(17.6 \%)\end{array}$ & $\begin{array}{c}8 \\
(47.1 \%)\end{array}$ & $\begin{array}{c}6 \\
(35.3 \%)\end{array}$ & 17 \\
\hline 24 & $\begin{array}{l}\text { Integrating Transit and } \\
\text { Urban Form }\end{array}$ & Concas & BD549-37 & $\begin{array}{c}2 \\
(11.8 \%)\end{array}$ & $\begin{array}{c}12 \\
(70.6 \%)\end{array}$ & $\begin{array}{c}3 \\
(17.6 \%)\end{array}$ & 17 \\
\hline 25 & $\begin{array}{l}\text { Development of Large } \\
\text { Bus/Small Bus Decision } \\
\text { Support Tool }\end{array}$ & Reich & BD549-39 & $\begin{array}{c}3 \\
(17.6 \%)\end{array}$ & $\begin{array}{c}11 \\
(64.7 \%)\end{array}$ & $\begin{array}{c}3 \\
(17.6 \%)\end{array}$ & 17 \\
\hline 26 & $\begin{array}{l}\text { Development of NTD } \\
\text { Tool for Vanpool } \\
\text { Services }\end{array}$ & Chu & BD549-40 & $\begin{array}{c}1 \\
(5.9 \%)\end{array}$ & $\begin{array}{c}14 \\
(82.4 \%)\end{array}$ & $\begin{array}{c}2 \\
(11.8 \%)\end{array}$ & 17 \\
\hline 27 & $\begin{array}{l}\text { Impacts of More } \\
\text { Rigorous ADA } \\
\text { Paratransit Eligibility } \\
\text { Assessments on Riders } \\
\text { with Disabilities }\end{array}$ & Sapper & BD549-44 & $\begin{array}{c}2 \\
(11.8 \%)\end{array}$ & $\begin{array}{c}10 \\
(58.8 \%)\end{array}$ & $\begin{array}{c}5 \\
(29.4 \%)\end{array}$ & 17 \\
\hline 28 & $\begin{array}{l}\text { Enhancing Transit } \\
\text { Safety and Security with } \\
\text { Wireless Detection and } \\
\text { Communication } \\
\text { Technologies }\end{array}$ & Barbeau & BD549-45 & $\begin{array}{c}5 \\
(31.3 \%)\end{array}$ & $\begin{array}{c}9 \\
(56.3 \%)\end{array}$ & $\begin{array}{c}2 \\
(12.5 \%)\end{array}$ & 16 \\
\hline 29 & $\begin{array}{l}\text { Toolbox for Transit } \\
\text { Event Investigation }\end{array}$ & Sapper & BD549-22 & $\begin{array}{c}6 \\
(33.3 \%)\end{array}$ & $\begin{array}{c}9 \\
(50.0 \%)\end{array}$ & $\begin{array}{c}3 \\
(16.7 \%)\end{array}$ & 18 \\
\hline 30 & $\begin{array}{l}\text { Moving the Bus Back } \\
\text { Into Traffic Safety }\end{array}$ & Lin & BD549-34 & $\begin{array}{c}6 \\
(33.3 \%)\end{array}$ & $\begin{array}{c}9 \\
(50.0 \%)\end{array}$ & $\begin{array}{c}3 \\
(16.7 \%)\end{array}$ & 18 \\
\hline
\end{tabular}

Figure C.1 provides a summary of the range of the report utilization frequency. Six of the NCTR reports were utilized by at least 30 percent of survey respondents while two-thirds of NCTR reports were utilized by fewer than 20 percent of survey respondents. No single report was utilized by more than 40 percent of survey respondents. 


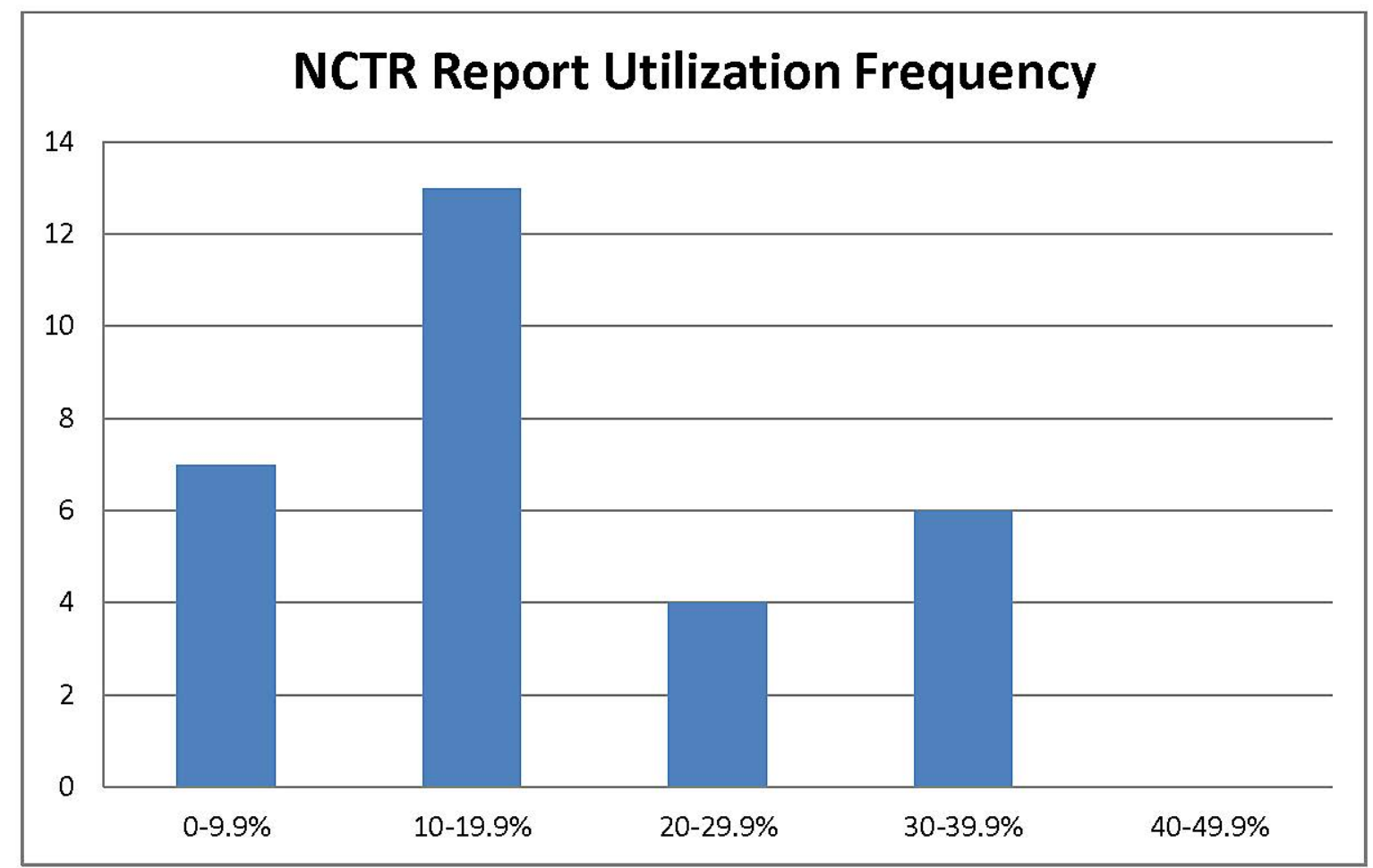

Figure C.1: NCTR Report Utilization Frequency

Question 2 of the survey asked survey respondents who had answered "yes" to Question 1 to describe how they had used the research results or tools.

Eight of the 18 survey respondents (44.4\%) provided input to this question. Examples of how survey respondents had utilized the research results or tools included:

- Revised practices to deliver improved service with accent on safety

- Used some of the info in our safety committee meetings.

- Reduced operating costs

- Used some the ideas to help establish project opportunities within our system

- We consistently review research studies, white papers and synthesis for potential improvements of our system(s).

- Reviewed the tools and implemented the ones that better fit organization goals and objectives.

- Provided further insight into practices, methods, or theory which has been used in the assessment or incorporation of the study's findings.

Question 3 of the survey asked survey respondents to specify what benefits they or their organization had realized from utilization of the research findings. Six of the 18 respondents (33.33\%) provided responses to this question, although most of the responses described the benefits in general terms and lacked specificity. Responses to Question 3 included: 
- We are going to decrease our work related injuries and accidents.

- Created more awareness of services.

- Optimized our workforce, "doing more with less."

- Added value to the conversation and collaboration with our [transit agency] partners.

- Benefits are difficult to attribute to the implementation of research findings.

- Improved planning, safer operations, better deliberation on relevant issues.

Question 4 captured respondents' overall level of satisfaction with the research reports, products and tools provided by CUTR/NCTR. The survey instrument utilized a 7-point scale and allowed respondents to express their satisfaction as "completely satisfied," "mostly satisfied," "somewhat satisfied," "neither satisfied nor dissatisfied," "somewhat dissatisfied," "mostly dissatisfied," or "completely dissatisfied."

Seventy-five percent (75\%) of survey respondents expressed some level of satisfaction with the research reports, products, and tools provide by CUTR/NCTR, with 16.67 percent expressing complete satisfaction. No survey respondents expressed any level of dissatisfaction, and 33.33 percent of respondents expressed ambivalence (neither satisfied nor dissatisfied).

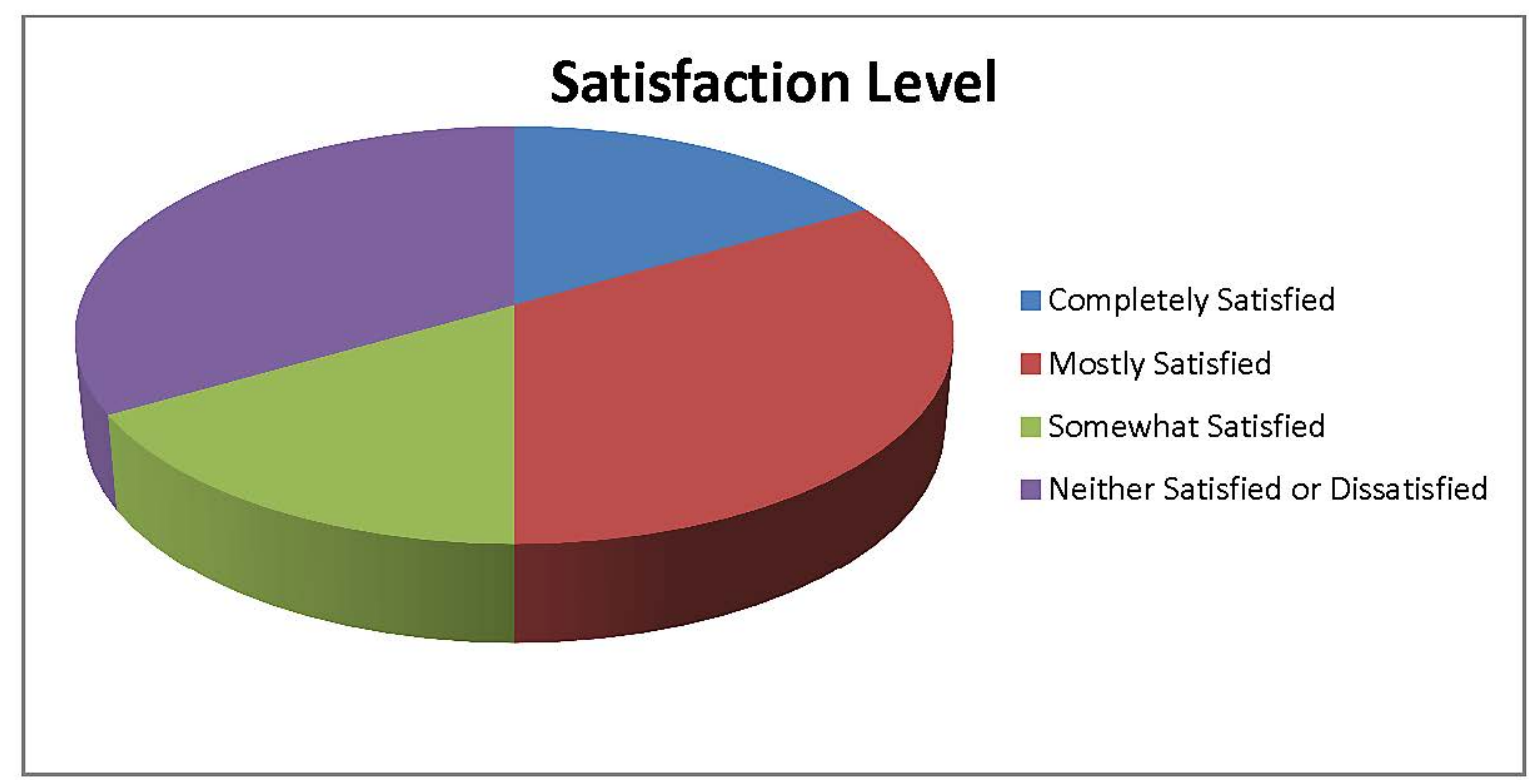

Figure C.2: Satisfaction Level

Question 5 afforded respondents the opportunity to augment their survey responses by asking respondents to provide information that would allow the report author to personally contact respondents. Five of the survey respondents provided contact information. The author attempted to telephonically contact each of the five survey respondents.

Following is a summary of insights provided by the three respondents with whom the author was able to visit: 
- John Ramos, Broward County Transit. Mr. Ramos' work with short range transit planning processes benefitted from NCTR research findings. He referenced NCTR research in the long and short range plans he prepared because it added credibility.

- Lina Kulikowski, Broward County Transit. Ms. Kulikowski was unaware of the NCTR reports. She is involved in a fare payment project and found NCTR Report BD54951, "Regional Fare Policy and Fare Allocation, Innovations in Fare Equipment and Data Collection" particularly valuable and intended to contact the report's Principal Investigator. Ms. Kulikowski was looking for ways to become informed of the availability of NCTR products and recommended Twitter as an appropriate dissemination tool.

- Sarah Perch, Manatee County Area Transit. Ms. Perch used the online survey as a reason to review several of the 30 NCTR research reports and now has a better understanding of the work NCTR conducts. She would appreciate executive summaries being emailed directly to her upon report publication.

\section{Discussion}

The findings from this task mirrored those documented in NCTR Project BDK85 \#977-30. In some instances, the data provided answers to the research questions and revealed high levels of customer awareness. In other instances, the data collection failed to provide answers to the research questions. This "failure" was primarily a function of the nature of the research and the degree to which results were promoted throughout the industry. Quantitative data did not directly provide answers to the research questions, but did offer indirect evidence, particularly to research question one. Qualitative data was limited and revealed wide variation with respect to project awareness and project utilization. 